\title{
mTOR-mediated podocyte hypertrophy regulates glomerular integrity in mice and humans
}

Victor G. Puelles, ${ }^{1,2,3,4}$ James W. van der Wolde, ${ }^{1}$ Nicola Wanner, ${ }^{4}$ Markus W. Scheppach, ${ }^{5}$ Luise A. Cullen-McEwen, ${ }^{1}$ Tillmann Bork, ${ }^{5}$ Maja T. Lindenmeyer, ${ }^{4}$ Lukas Gernhold, ${ }^{4}$ Milagros N. Wong, ${ }^{4}$ Fabian Braun, ${ }^{4}$ Clemens D. Cohen, ${ }^{6}$ Michelle M. Kett, ${ }^{7}$ Christoph Kuppe, ${ }^{8}$ Rafael Kramann, ${ }^{8}$ Turgay Saritas, ${ }^{8}$ Claudia R. van Roeyen, ${ }^{8}$ Marcus J. Moeller, ${ }^{8}$ Leon Tribolet, ${ }^{1}$ Richard Rebello, ${ }^{1}$ Yu B.Y. Sun, ${ }^{1}$ Jinhua Li, ${ }^{1}$ Gerhard Müller-Newen, ${ }^{9}$ Michael D. Hughson, ${ }^{10}$ Wendy E. Hoy, ${ }^{11}$ Fermin Person, ${ }^{12}$ Thorsten Wiech, ${ }^{12}$ Sharon D. Ricardo, ${ }^{1}$ Peter G. Kerr, ${ }^{2,3}$ Kate M. Denton, ${ }^{7}$ Luc Furic, ${ }^{13,14,15}$ Tobias B. Huber, ${ }^{4}$ David J. Nikolic-Paterson, ${ }^{2,3}$ and John F. Bertram

'Development and Stem Cells Program, Monash Biomedicine Discovery Institute and Department of Anatomy and Developmental Biology, Monash University, Melbourne, Australia. 'Department of Nephrology, Monash Health, Melbourne, Australia. ${ }^{3}$ Center for Inflammatory Diseases, Monash University, Melbourne, Australia. ${ }^{4}$ III. Department of Medicine, University Medical Center Hamburg-Eppendorf, Hamburg, Germany. ${ }^{5}$ Renal Division, University Medical Center Freiburg, Freiburg, Germany. ${ }^{6}$ Nephrological Center Medical Clinic and Polyclinic IV, University of Munich, Munich, Germany. ${ }^{7}$ Cardiovascular Program, Monash Biomedicine Discovery Institute and Department of Physiology, Monash University, Melbourne, Australia. ${ }^{8}$ Department of Nephrology and Clinical Immunology and 'Institute of Biochemistry and Molecular Biology, RWTH Aachen University, Aachen, Germany. ${ }^{10}$ Department of Pathology, University of Mississippi Medical Center, Jackson, Mississippi, USA. "Centre for Chronic Disease, The University of Queensland, Brisbane, Queensland, Australia. ${ }^{12}$ Institute of Pathology, University Medical Center Hamburg-Eppendorf, Hamburg, Germany. ${ }^{13}$ Prostate Cancer Translational Research Laboratory, Peter MacCallum Cancer Centre ${ }^{14} \mathrm{Sir}$ Peter MacCallum Department of Oncology, University of Melbourne, Australia. ${ }^{15}$ Cancer Program, Biomedicine Discovery Institute and Department of Anatomy and Developmental Biology, Monash University, Melbourne, Australia.

The cellular origins of glomerulosclerosis involve activation of parietal epithelial cells (PECs) and progressive podocyte depletion. While mammalian target of rapamycin-mediated (mTORmediated) podocyte hypertrophy is recognized as an important signaling pathway in the context of glomerular disease, the role of podocyte hypertrophy as a compensatory mechanism preventing PEC activation and glomerulosclerosis remains poorly understood. In this study, we show that glomerular mTOR and PEC activation-related genes were both upregulated and intercorrelated in biopsies from patients with focal segmental glomerulosclerosis (FSCS) and diabetic nephropathy, suggesting both compensatory and pathological roles. Advanced morphometric analyses in murine and human tissues identified podocyte hypertrophy as a compensatory mechanism aiming to regulate glomerular functional integrity in response to somatic growth, podocyte depletion, and even glomerulosclerosis - all of this in the absence of detectable podocyte regeneration. In mice, pharmacological inhibition of mTOR signaling during acute podocyte loss impaired hypertrophy of remaining podocytes, resulting in unexpected albuminuria, PEC activation, and glomerulosclerosis. Exacerbated and persistent podocyte hypertrophy enabled a vicious cycle of podocyte loss and PEC activation, suggesting a limit to its beneficial effects. In summary, our data highlight a critical protective role of mTOR-mediated podocyte hypertrophy following podocyte loss in order to preserve glomerular integrity, preventing PEC activation and glomerulosclerosis.

exists.

Copyright: (c) 2019, American Society for Clinical Investigation.

Submitted: December 20, 2017

Accepted: August 8, 2019

Published: September 19, 2019.

Reference information: /CI Insight. 2019;4(18):e99271.

https://doi.org/10.1172/jci.

insight.99271.

\section{Introduction}

Close to $13 \%$ of the adult world population suffers from some form of chronic kidney disease (CKD) (1). The high social, economic, and mortality burden of CKD highlights the urgent need for a better understanding of its pathophysiology in the search for new and effective therapies (2). Approximately $90 \%$ of kidney diseases leading to CKD originate in the kidney filters (glomeruli) (3). For this reason, glomerular resident cells are important targets of current research. 
Podocytes are glomerular epithelial cells that cover the filtration surface area and serve as master regulators of kidney function (4). As terminally differentiated cells, podocytes cannot reenter the cell cycle to complete cytokinesis, limiting their capacity for self-renewal (5). Wharram et al. (6) showed that podocyte loss is sufficient to cause glomerulosclerosis, leading to progressive loss of kidney function and, thereby, CKD. Importantly, humans lose podocytes during normal aging (7) and exhibit more extensive podocyte loss in association with the leading causes of CKD, including diabetes $(8,9)$. Therefore, podocyte depletion is considered a unifying principle of glomerular disease (3).

Glomerulosclerosis develops after podocyte depletion has reached a critical threshold that is still inaccurately defined (approximately $20 \%-40 \%$ podocyte loss) $(6,10,11)$. Recent evidence shows that parietal epithelial cells (PECs), glomerular resident cells that are part of Bowman's capsule, play key roles in the development of glomerulosclerotic lesions. PECs can become activated in a process defined by increased potential for migration to the glomerular tuft, prominent proliferation, abnormal production of extracellular matrix, and de novo expression of the cell surface marker CD44 (12-14). However, the interaction between podocyte depletion and PEC activation remains poorly understood.

When podocyte depletion does not reach the critical threshold for disease, transient acute albuminuria is followed by clinical recovery without overt permanent structural changes $(6,11)$, suggesting that low levels of podocyte loss trigger an adaptive response in remaining podocytes that is able to preserve glomerular integrity. It has been hypothesized that remaining podocytes undergo hypertrophy in order to recover the filtration surface area, at least until the degree of stress is no longer tolerable (15). However, the lack of reliable methods for the accurate and precise analysis of podocyte number and size has limited the study of podocyte hypertrophy as an adaptive response in vivo.

The mammalian target of rapamycin complex 1 (mTORC1) signaling pathway, a key regulator of cell growth, is essential for normal nephrogenesis (16-18). In contrast, hyperactivity of mTOR signaling in podocytes plays a central role in the development of diabetic nephropathy (19) and crescentic nephritis (20). mTOR inhibition has proven to be an effective therapeutic approach in animal models of glomerular disease and in small clinical studies (16), but it has also been associated with development and exacerbation of proteinuria and glomerulosclerosis (21-27). Recent studies by Zschiedrich, et al. (28), and Nishizono, et al. (29), have shown that ablation/inhibition of mTORC1 in podocytes resulted in an increased vulnerability toward glomerulosclerosis in both mice and rats, raising questions about the role of mTORC1 signaling as an important adaptive mechanism in podocytes.

Our current study shows that mTOR-mediated podocyte hypertrophy is required to sustain glomerular integrity following podocyte loss, suggesting that podocyte hypertrophy prevents the development of PEC activation and glomerulosclerosis in the absence of podocyte regeneration. Importantly, our findings also indicate that exacerbated mTOR-mediated podocyte hypertrophy is connected to a vicious cycle of progressive podocyte loss that also results in PEC activation and glomerulosclerosis. Thus, podocyte hypertrophy provides a classical example of a compensatory response that can become maladaptive with time and persistent damage.

\section{Results}

Upregulation of $m$ TOR and PEC activation-related genes in human FSGS. Glomerular extracts from biopsies in patients with primary focal segmental glomerulosclerosis (FSGS) showed an upregulation of genes underlying mTOR signaling in comparison with living donors with mTORC1 signaling being upregulated in podocytes, as shown by phosphorylation of ribosomal protein S6 (p-rp-S6), a downstream target of mTORC1 directly associated with cell size regulation (Figure 1A). Glomerular extracts from biopsies in patients with primary FSGS showed an upregulation of PEC activation-related genes in comparison with living donors with PEC activation marker CD44, a cell surface glycoprotein that is expressed de novo by activated PECs, being identified in serial sections where histological identification of a segmental lesion based on periodic acid-Schiff (PAS) and expression of CD44 in the same area were confirmed (Figure 1B). While all markers of PEC activation were associated with at least 1 mTOR-related gene, the best correlations were found using CD9, CD44, CXCR4, and KRT19 (Figure 1C). These associations suggest that PEC activation and podocyte hypertrophy may have direct interactions in the development of human FSGS

Human podocyte hypertrophy during somatic growth and FSGS. First, we aimed to quantify the degree of podocyte depletion and hypertrophy in humans (Supplemental Tables 1-3 contain demographic data from included subjects; supplemental material available online with this article; https://doi.org/10.1172/jci.insight.99271DS1). A combination of indirect immunofluorescence, confocal microscopy, and model-based stereology was applied in 
A

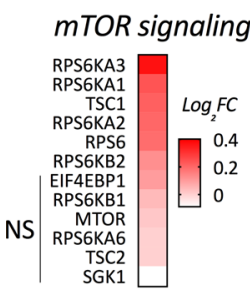

B



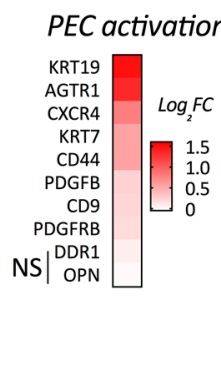

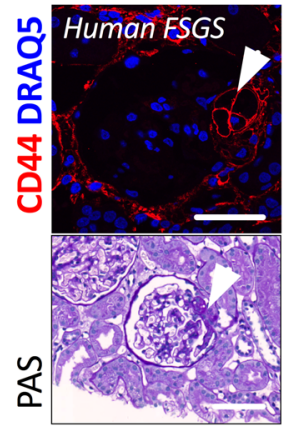

C

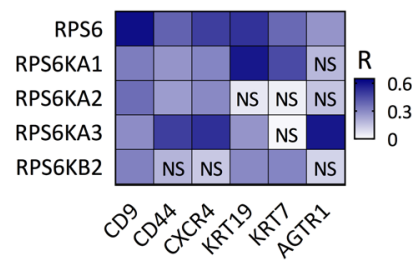

D

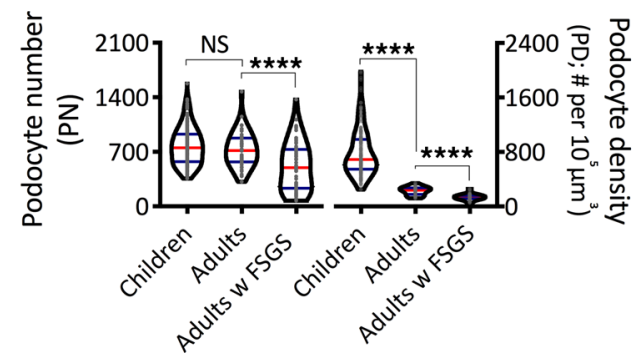

E
$\mathbf{F}$

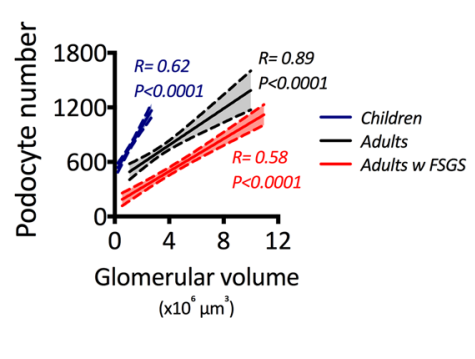

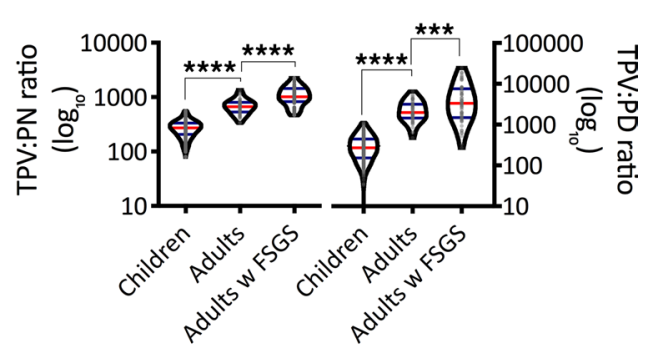

Figure 1. mTOR-mediated podocyte hypertrophy as a compensatory response to human somatic growth and FSGS. (A) Transcriptional regulation of mTOR signaling in glomerular extracts from human biopsies. Comparison between FSCS $(n=23)$ and living donors $(n=42)$, showing fold-change of each respective gene ( $q<0.05$ in all genes except those with NS), and representative confocal image from indirect immunofluorescence showing a podocyte specific marker (Wilms' Tumor 1; WT-1) and a downstream target of mTORC1 (phosphorylated ribosomal protein S6; p-rp-S6) in a glomerulus from a patient with primary FSCS. Scale bar: $10 \mu \mathrm{m}$. (B) Transcriptional regulation of PEC activation-related genes in glomerular extracts from human biopsies. Comparison between FSGS $(n=23)$ and living donors $(n=42)$, showing fold-change of each respective gene $(q<0.05$ in all genes except those with no significance [NS]) and representative confocal image of PEC activation marker CD44, and histological staining (periodic acid-Schiff; PAS) showing sequential physical sections of a glomerulus with a segmental lesion (arrowhead). Scale bars: $100 \mu \mathrm{m}$. (C) Correlation analysis of regulated mTOR signaling and PEC activation genes. Each box represents an independent association (spearman rank coefficient; $R$ ). Every association was statistically significant unless labeled with NS. (D) Comparison of podocyte depletion indices (podocyte number, PN; podocyte density, PD) between glomeruli from children, adults, and adults with FSCS. (E) Association between podocyte number and glomerular volume (using Spearman's rank coefficients) among children (blue), adults (gray), and adults with FSCS (red). Solid lines represent the regression line, and dotted lines the 95\% CI. (F) Comparison of podocyte size indices (total podocyte volume divided by podocyte number, TPV:PN ratio; and total podocyte volume divided by podocyte density, TPV:PD ratio) between children, adults, and adults with FSCS. ${ }^{* * *} P<0.0001 ;{ }^{* * *} P<0.001$. In violin plots, red lines represent medians and blue lines represent IQRs; every gray dot represents 1 glomerulus. Kruskal-Wallis with Dunn's multiple comparisons tests were used.

advanced podocyte morphometrical analyses (podometrics), including glomerular size, podocyte number, podocyte density, and ratios marking podocyte size (total podocyte volume per podocyte number, TPV:PN; and total podocyte volume per podocyte density, TPV:PD) in autopsied kidneys from children, nephrectomy samples from adults without kidney disease, and human biopsies from adult patients with FSGS.

While there was no statistical reduction in the number of podocytes between children and adults, there was a strong decrease in podocyte density $(P<0.0001)$. Podocyte number and density were significantly reduced in adult patients with primary FSGS compared with adult controls $(P<0.0001$; Figure 1D). There was a strong correlation between podocyte number and glomerular size in all 3 groups (Figure 1E). While the slopes were significantly different between children and adults $(P<0.0001)$, the slopes between adults and FSGS patients were identical, but the elevations were significantly different $(P<0.0001)$. Podocyte size showed graded increases from childhood to adulthood and then to adults with primary FSGS $(P<0.001$; Figure $1 \mathrm{~F}$ ). Together, the data suggest postnatal podocyte loss and hypertrophy across the full spectrum of glomerular sizes in the adult kidney, a process that is exacerbated in the context of FSGS.

Interestingly, podocyte densities and sizes from adults without kidney disease and patients with FSGS showed significant overlap (Supplemental Figure 1, A and B). Furthermore, the percentage of glomeruli from FSGS patients was higher in the top quintiles of podocyte density (Q1 with the lowest values for podocyte density; Supplemental Figure 1C) and size (Q5 with the highest values of TPV:PD; Supplemental Figure 1D), reinforcing the concept that podocyte depletion and hypertrophy are closely associated. 
A

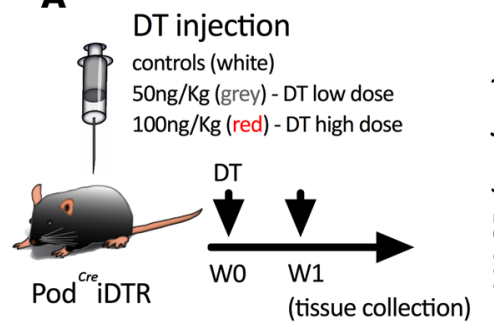

B

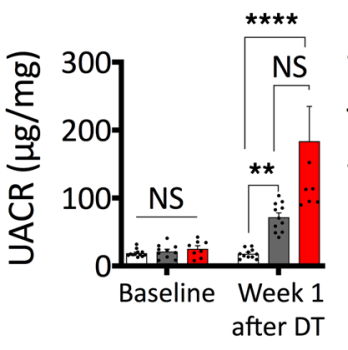

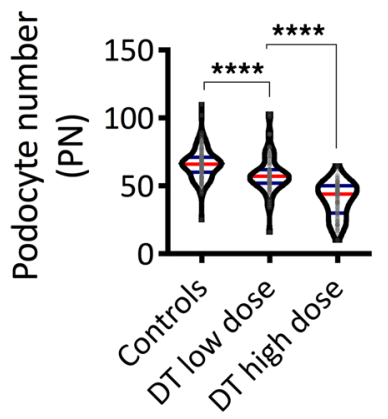

E

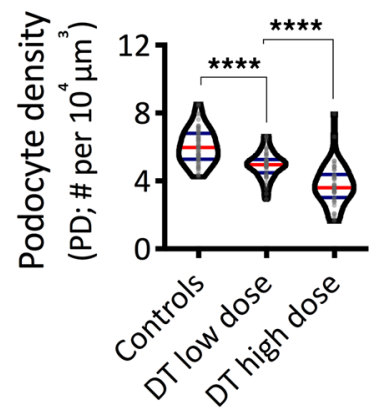

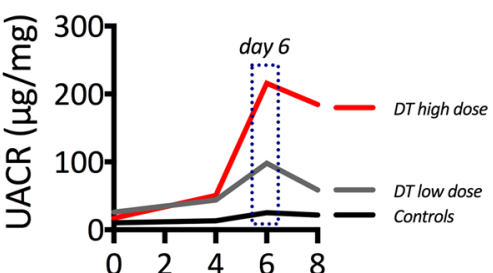

Days after DT injection
C



\section{H mTORC1 activity after acute podocyte loss}

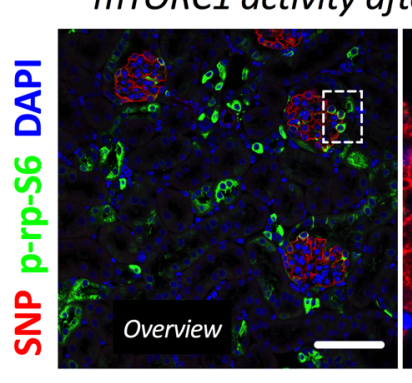





$\mathbf{F}$

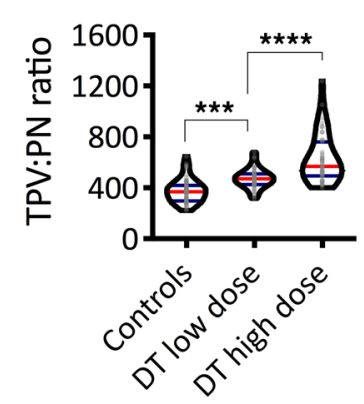

G

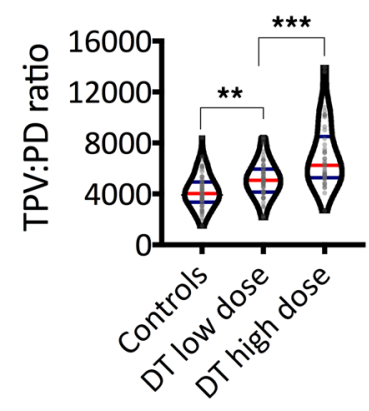

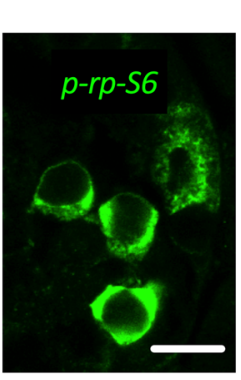

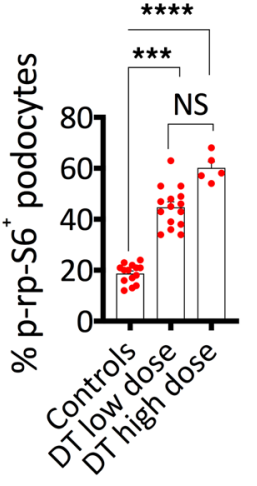

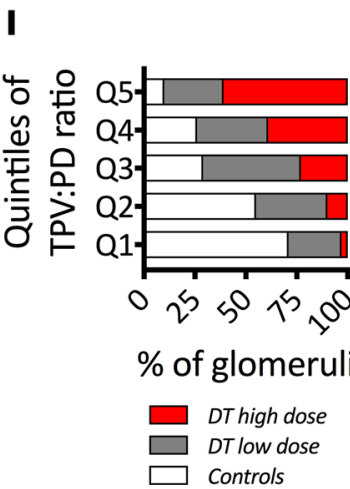

Figure 2. mTOR-mediated podocyte hypertrophy during acute podocyte loss. (A) Schematic representation of experimental design. (B) Urinary albumin to creatinine ratio (UACR); on the left, we show average per time point, and on the right, the evolution every 2 days per group. (C) Three-dimensional reconstruction of an intact mouse glomerulus after indirect immunofluorescence, solvent-based optical clearing, and confocal microscopy showing double labeling of podocytes with p57 (red) and synaptopodin (SNP, green). (D) Podocyte number. (E) Podocyte density. (F) Total podocyte volume per unit of podocyte number (TPV:PN ratio). (G) Total podocyte volume per unit of podocyte density (TPV:PD ratio). (H) Representative confocal image showing indirect immunofluorescence of a podocyte marker (SNP, red) and a downstream target of mTORC1 (phosphorylated ribosomal protein S6, p-rp-S6 in green) and quantification of the percentage of $\mathrm{p}$-rp-S6-positive podocytes per group. (I) Quintile analysis of TPV:PD. ${ }^{* * * *} P<0.0001$; ${ }^{* *} P<0.001$; ${ }^{* *} P<0.01$. In B, bars represent means and error bars \pm SEMs. In violin plots, red lines represent medians and blue lines represent IQRs; every gray dot represents 1 glomerulus. Kruskal-Wallis with Dunn's multiple comparisons tests were used. Scale bars: (C) $30 \mu \mathrm{m}$, (H overview) $70 \mu \mathrm{m}$, and (H panels) $10 \mu \mathrm{m}$.

Mouse podocyte hypertrophy during somatic growth and FSGS. Given that human samples do not allow the study of dynamic cellular changes, we utilized a mouse model of selective podocyte depletion based on the expression of a mutated human diphtheria toxin (DT) receptor under a podocyte-specific promoter (Pod ${ }^{\text {Cre }}$ iDTR). Noninjected mice showed a similar response to somatic growth as humans - as body size increased (Supplemental Figure 2A), compensatory glomerular hypertrophy (Supplemental Figure 2B) led to reductions in podocyte density (Supplemental Figure 2C), which resulted in elevations in podocyte size (Supplemental Figure 2D), confirming a similar response in mice and humans.

Pod ${ }^{\text {Cre }}$ iDTR were injected either a low dose $(50 \mathrm{ng} / \mathrm{kg})$ or a high dose $(100 \mathrm{ng} / \mathrm{kg})$ of DT. Mice were continuously monitored via urinary albumin to creatinine ratios (UACRs) every 2 days. Experimental mice were sacrificed 1 week after DT injection, and tissue was collected for cellular analyses (Figure 2A). 
A

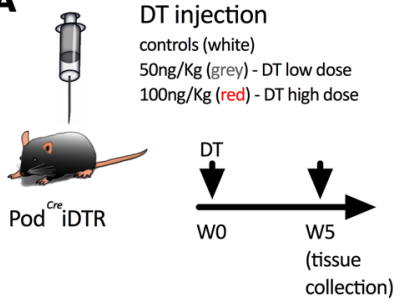

B

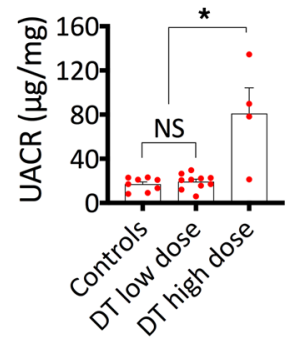

C
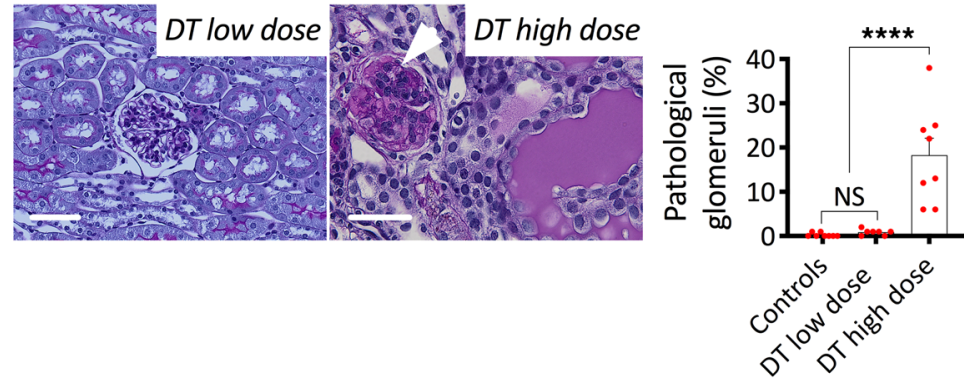

$\mathbf{E}$

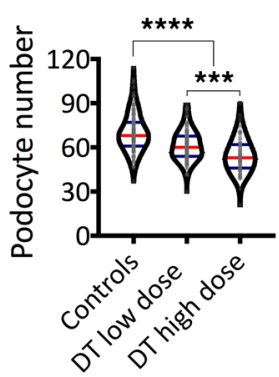

$\mathbf{F}$

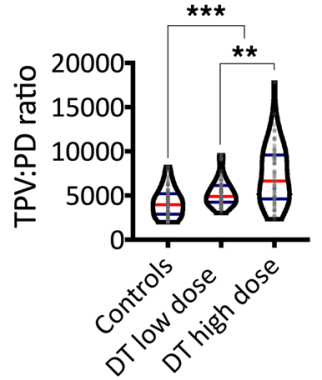

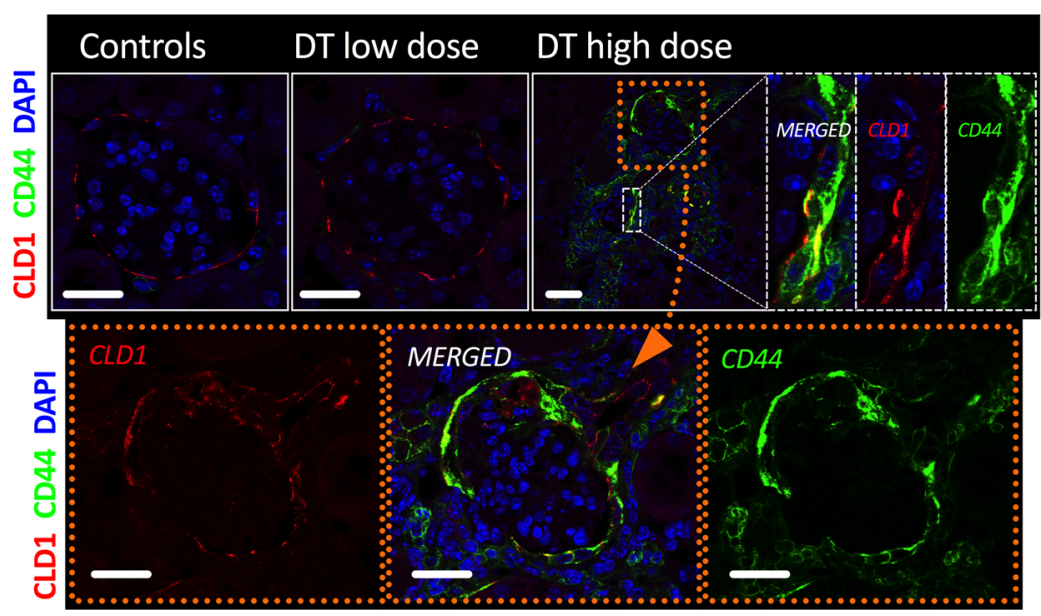

Figure 3. mTOR-mediated podocyte hypertrophy in a mouse model of FSCS. (A) Schematic representation of experimental design. (B) Urinary albumin to creatinine ratio (UACR). (C) Periodic acid-Schiff (PAS) histological staining showing glomerulosclerosis in mice injected with high dose of DT and quantification of pathological glomeruli (\%). (D) Representative confocal images after indirect immunofluorescence of a PEC-specific marker (Claudin-1; CLD1) and a PEC activation marker (CD44). (E) Podocyte number. (F) Total podocyte volume per unit of podocyte density (TPV:PD ratio). ${ }^{* * *} P<0.0001 ;{ }^{* *} P<0.001$; ${ }^{* *} P<0.01$; ${ }^{*} P<0.05$. In B, bars represent means and error bars \pm SEMs. Each dot represents 1 mouse. In violin plots, red lines represent medians and blue lines represent IQRs; every gray dot represents 1 glomerulus. Kruskal-Wallis with Dunn's multiple comparisons tests were used. Scale bars: (C) $50 \mu \mathrm{m}$, (D) $30 \mu \mathrm{m}$.

While we observed a graded increase in albuminuria based on DT dose, the peak of albuminuria was identified around the same time point (day 6 after DT) in both groups (Figure 2B)

A representative 3-dimensional (3-D) reconstruction of an intact mouse glomerulus is provided as an example of our approach for advanced podometrics, which combines indirect immunofluorescence, solvent-based optical clearing, laser confocal microscopy, and 3-D bioinformatics (Figure 2C). Our results mirrored our findings in humans, as podocyte loss directly correlated with increases in podocyte size (Figure 2, D-G). As in humans, we observed a strong upregulation of mTORC1 in podocytes after acute podocyte loss (Figure $2 \mathrm{H}$ ).

Quintile analysis of podocyte size confirmed the strong association between podocyte loss and hypertrophy, and it showed that even the highest quintile of podocyte size (Q5) contained glomeruli from control mice (Figure 2I), highlighting the concept of physiological mTOR-mediated podocyte hypertrophy.

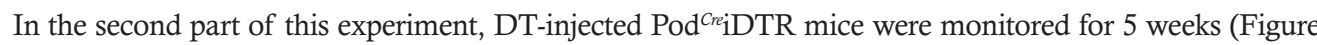
$3 \mathrm{~A})$. By the end of the follow-up period, albuminuria resolved in mice injected with a low dose of DT and persisted in mice injected with a high dose of DT (Figure 3B). Furthermore, glomerulosclerotic lesions (Figure 3C) and activated PECs (Figure 3D) were only observed in mice injected with a high dose of DT. Interestingly, podocyte loss (Figure 3E) and hypertrophy (Figure 3F) remained after 5 weeks of DT injection at both doses.

Glomerular functional recovery: podocyte hypertrophy versus podocyte regeneration. While albuminuria was

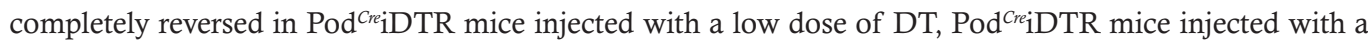
high dose of DT showed only partial resolution of albuminuria 5 weeks after DT injection (Supplemental Figure 3A). Podocyte hypertrophy was sustained between weeks 1 and 5 after DT injection at both doses (Supplemental Figure 3B). Surprisingly, podocyte number appeared to increase by $11 \%(P<0.0001)$ between weeks 1 and 5 only in mice injected with a high dose of DT (Supplemental Figure 3C) - a finding 
A

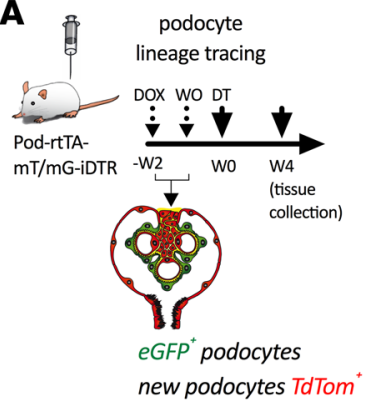

B

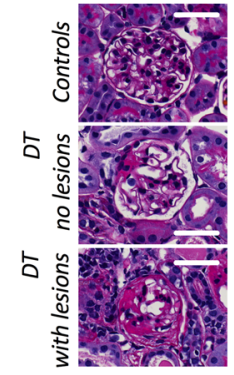

C

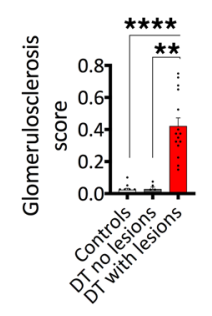

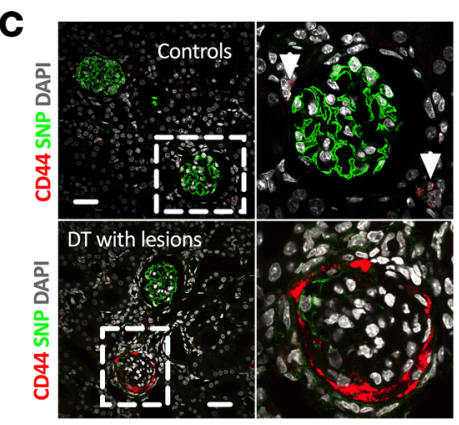

D Baseline d7-d10 week 4

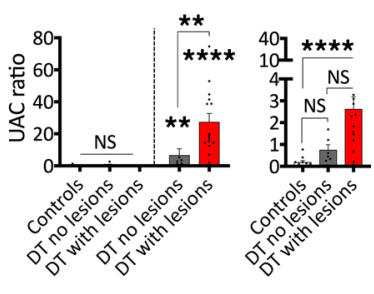

H



E
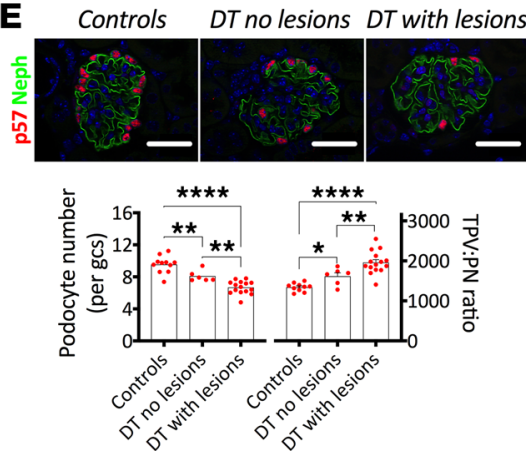

F
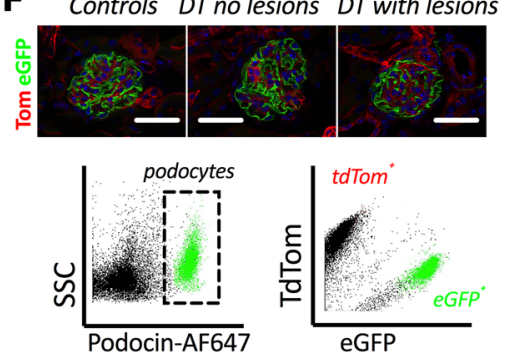

G

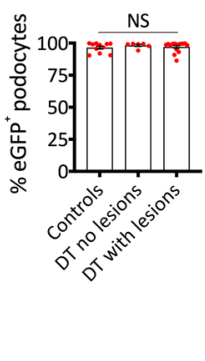

Figure 4. Glomerular functional recovery is not associated with podocyte regeneration in a mouse model of FSCS. (A) Schematic representation of experimental design, where a single diphtheria toxin (DT) injection is applied after oral doxycycline administration and a subsequent wash out (WO) period in order to genetically label podocytes with eGFP. (B) Representative images of glomerulosclerosis with quantitation of glomerulosclerosis scores. (C) De novo PEC activation indicated by upregulation of CD44 (red) in PECs using synaptopodin (SNP; green) as a podocyte marker. (D) Urinary albumin to creatinine ratio (UACR). (E) Progressive podocyte depletion and hypertrophy in glomeruli from transgenic mice injected with DT. (F) A combination of lineage tracing and immunolabeling allows FACS-based analysis of podocyte regeneration. (G) The percentage of GFP+ podocytes does not vary after induction of different levels of podocyte loss and development of FSGS. (H) Correlation between podocyte depletion and glomerulosclerosis scores. ${ }^{* * * *} P<0.0001$; ${ }^{* *} P<0.01 ;{ }^{*} P<0.05$. Bars represent means and error bars \pm SEMs. Kruskal-Wallis with Dunn's multiple comparisons tests were used. In correlation analyses (Spearman's rank coefficients), solid lines represent the regression line and dotted lines the $95 \% \mathrm{Cl}$. Scale bars: $30 \mu \mathrm{m}$.

that caught our attention, as it may suggest the possibility of podocyte regeneration. At week 1 after highdose DT injection, we could clearly identify podocytes with very negligible p57 nuclear expression (Supplemental Figure 3D). We quantified the percentage of synaptopodin-positive $\left(\mathrm{SNP}^{+}\right)$podocytes that coexpressed p57 in all groups (Supplemental Figure 3E), which revealed that, even at baseline, approximately $1.52 \%$ of podocytes had low or negligible levels of $\mathrm{p} 57$ expression. While a low dose of DT barely altered this percentage $(2.14 \%-2.20 \%)$, a high dose of DT led to a significant increase in $\mathrm{p} 57^{-}$podocytes at week 1 after DT injection (13.73\%) that returned to normal $(2.40 \%)$ by 5 weeks after DT injection, suggesting that the apparent increase in podocyte number most likely reflects reversible podocyte injury, where injured podocytes transiently downregulate expression of $\mathrm{p} 57$, which then returned to normal.

To further explore the possibility of podocyte regeneration in this model, we used a second transgenic mouse model for selective dose-dependent podocyte loss (Pod-rtTA-mT/mG-iDTR), which was based on a previous publication (30). Upon doxycycline administration, podocytes permanently expressed eGFP and a mutated DT receptor, whereas all other cells were labeled with TdTomato (Figure 4A) (10). After a wash out period, DT was injected at multiple doses (see Methods). Mice were stratified into 2 categories based on histopathology analysis into DT with and without glomerulosclerotic lesions (Figure 4B). This definition also correlated with the presence of activated PECs (Figure 4C) and albuminuria (Figure 4D). As expected, we observed graded podocyte loss and hypertrophy, which mirrored our previous experiments (Figure 4E). Glomeruli from 1 kidney of each mouse were enzymatically digested into a single cell suspension and immunolabeled with a podocyte-specific antibody (podocin). Podocin ${ }^{+}$podocytes were isolated using flow cytometry and divided into $\mathrm{eGFP}^{+}$(genetically labeled podocytes) and TdTomato ${ }^{+}$(fraction of nonlabeled podocytes; Figure $4 \mathrm{~F}$ ), which allowed the calculation of a percentage of $\mathrm{eGFP}^{+}$podocytes per mouse. Podocytes cannot reenter the cell cycle to undergo division (5), which means that the percentage of eGFP ${ }^{+}$ podocytes cannot expand due to podocyte mitosis. If podocyte regeneration were actively taking place, we 
A

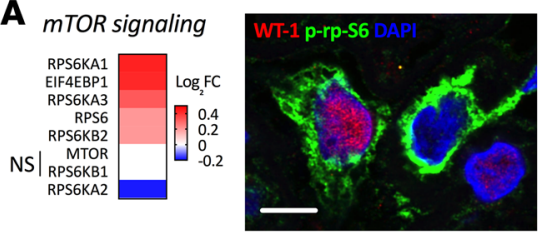

B



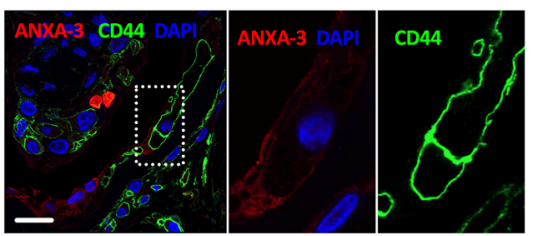

C

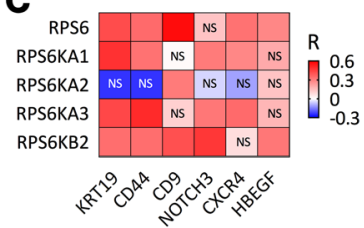

D
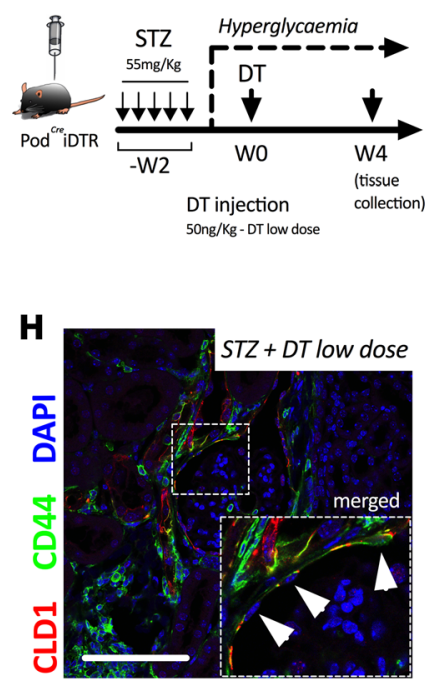

$\mathbf{E}$

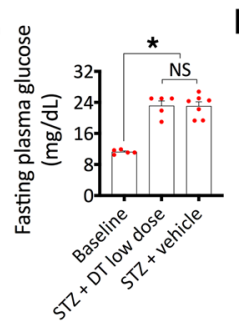

I

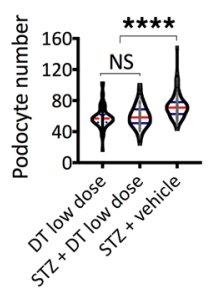

$\mathbf{F}$

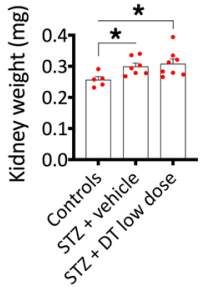

G

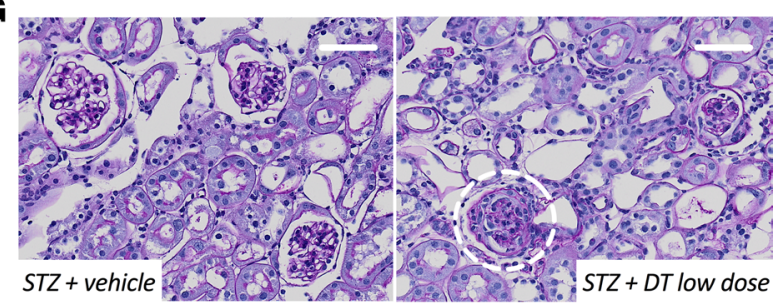

J

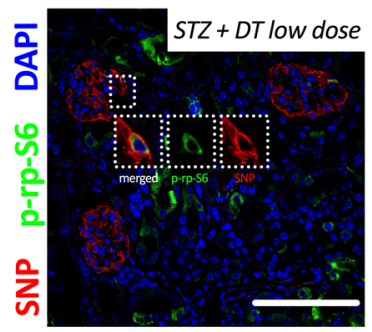

$\mathbf{K}$

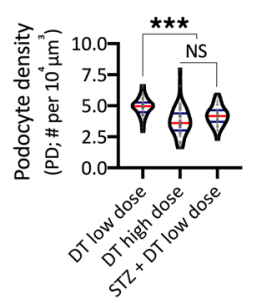

$\mathbf{L}$



Figure 5. mTOR-mediated podocyte hypertrophy in diabetic mice and humans. (A) Transcriptional regulation of mTOR signaling in glomerular extracts from human biopsies. Comparison between diabetic nephropathy (DN; $n=14)$ vs. living donors $(n=42)$, showing fold-change of each respective gene $(q<0.05$ in all genes except those with NS), and representative confocal image from indirect immunofluorescence showing a podocyte specific marker (Wilms' Tumor 1; WT-1) and a downstream target of mTORC1 (phosphorylated ribosomal protein S6; p-rp-S6) in a glomerulus from a patient with DN. Scale bar: $10 \mu \mathrm{m}$. (B) Transcriptional regulation of PEC activation-related genes in glomerular extracts from human biopsies. Comparison between DN $(n=14)$ vs. living donors $(n=42)$, showing fold-change of each respective gene $(q<0.05$ in all genes except those with NS) and representative confocal image of PEC activation marker CD44 (green) and PEC specific marker Annexin 3 (ANXA3; red). Scale bars: $10 \mu \mathrm{m}$. (C) Correlation analysis of regulated mTOR signaling and PEC activation genes. Each box represents an independent association (spearman correlation; $R$ ). Every association was statistically significant unless labeled with NS. (D) Schematic representation of experimental design for a hyperglycemic model via streptozotocin (STZ) injection with subsequent injection of diphtheria toxin (DT) to induce selective podocyte loss. (E) Fasting plasma glucose. (F) Kidney weight. (G) Periodic acidSchiff (PAS) stainings showing development of glomerular lesions only after DT injection. (H) De novo PEC activation (CD44 upregulation; green). (I) Podocyte number. (J) Representative confocal image showing upregulation of mTORC1 signaling in podocytes after podocyte loss. (K) Podocyte density. (L) Total podocyte volume by unit of podocyte density (TPV:PD ratio). ${ }^{* * *} P<0.0001 ;{ }^{* *} P<0.001 ;{ }^{*} P<0.05$. In $\mathbf{E}$ and $\mathbf{F}$, bars represent means and error bars \pm SEMs. Each dot represents 1 mouse. In violin plots, red lines represent medians and blue lines represent IQRs; every gray dot represents 1 glomerulus. Kruskal-Wallis with Dunn's multiple comparisons tests were used. Scale bars: (C) $50 \mu \mathrm{m}$, and (H and J) $100 \mu \mathrm{m}$.

would expect an expansion of the percentage of $\mathrm{TdTomato}^{+}$podocytes, which would directly reduce the percentage of $\mathrm{GGFP}^{+}$podocytes. However, we did not observe any changes in the percentage of $\mathrm{eGFP}^{+}$ podocytes in any of the groups (Figure $4 \mathrm{G}$ ), and numbers of podocytes per glomerular cross-section had a strong inverse correlation with glomerulosclerosis (Figure $4 \mathrm{H}$ ). Together, these findings provide strong evidence that sustained podocyte hypertrophy plays a central role in functional glomerular recovery in the absence of detectable podocyte regeneration.

Podocyte hypertrophy in diabetic mice and humans. Next, we focused on a mouse model that combines podo-

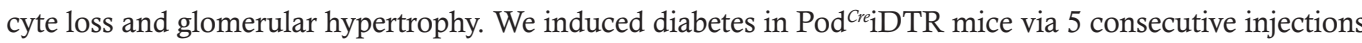
of streptozotocin (STZ; Supplemental Figure 4A). Two weeks after the first STZ injection, plasma blood glucose was significantly elevated (Supplemental Figure 4B). After 4 weeks of persistent hyperglycemia, STZ-injected mice developed mild albuminuria (Supplemental Figure 4C). Six weeks after the first STZ injection, mice developed glomerular hypertrophy (Supplemental Figure 4D), reductions in podocyte number and density (Supplemental Figure 4, E and F), and increases in podocyte size (Supplemental Figure 4G).

Human diabetic nephropathy shared a similar transcriptional profile as FSGS. Glomerular extracts from biopsies in patients with diabetic nephropathy showed an upregulation of mTOR signaling and PEC activation-related genes in comparison with living donors (Figure 5, A and B). P-rp-S6 was identified in 
podocytes (Figure 5A), and PEC activation marker CD44 was detected in scattered PECs (Figure 5B). While all markers of PEC activation were associated with at least 1 mTOR-related gene, the best correlations were found for KRT19 and CD44 (Figure 5C).

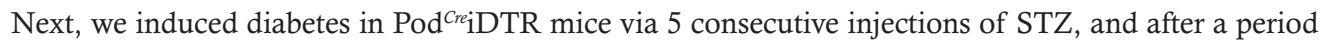
of 2 weeks to verify hyperglycemia, we injected low-dose DT (Figure 5D). Diabetic mice achieved the same level of hyperglycemia in both groups (DT-injected vs. vehicle-injected controls; Figure 5E). Diabetes led to significant increases in kidney weight (Figure 5F), a surrogate marker of renal hyperfiltration. However, only diabetic mice injected with low-dose DT developed glomerulosclerotic lesions (Figure 5G) and PEC activation (Figure $5 \mathrm{H}$ ) within 4 weeks after DT injection, despite losing the same number of podocytes as nondiabetic mice injected with a low dose of DT (Figure 5I). As in human biopsies, mTORC1 signaling was also upregulated in podocytes from diabetic mice injected with DT (Figure 5J). Reductions in podocyte density were comparable with those observed in mice injected with a high dose of DT (Figure $5 \mathrm{~K}$ ), as glomerular hypertrophy in diabetic mice acted as a second stressor. The exacerbated level of podocyte hypertrophy driven by podocyte loss and glomerular hypertrophy was comparable with FSGS induced by a high dose of DT (Figure 5L) and was not sufficient to prevent PEC activation or glomerulosclerosis. Together, these findings suggest that the degree of podocyte depletion marks the limits of compensatory podocyte hypertrophy and, thereby, preservation of glomerular integrity.

Exacerbated mTOR hyperactivation in mouse podocytes leads to PEC activation and FSGS. Tuberous sclerosis complex 1 (TSC1) is a well-known repressor of mTORC1 signaling, with its genetic deletion leading to a direct and persistent hyperactivation of $\operatorname{mTORC1}$ (19). In this experiment, we analyzed tissue collected between weeks 4 and 16 of age from Pod-TSC1 ${ }^{-/-}$mice, where mTORC1 hyperactivation was confirmed in isolated podocytes (Figure 6A). Representative confocal images show clear increases in podocyte size and de novo PEC activation in Pod-TSC $1^{-/-}$mice, where CD44+ $\mathrm{PECs}$ were observed in $50 \%$ of Pod-TSC $1^{-/-}$ mice by $4-6$ weeks of age and $100 \%$ of Pod-TSC1 $1^{-/}$mice by $12-16$ weeks of age (Figure $6 \mathrm{~B}$ ). Four- to 6-week-old Pod-TSC1 ${ }^{-/-}$mice showing PEC activation had lower numbers of podocytes (Supplemental Figure 5A) and higher glomerular volumes (Supplemental Figure 5B) than Pod-TSC1 ${ }^{-1-}$ mice without PEC activation, suggesting that accelerated podocyte loss in the context of exacerbated glomerular hypertrophy play central roles in the development of PEC activation.

Between 4 and 16 weeks of age, Pod-TSC1 $1^{-/-}$mice showed progressive glomerular hypertrophy (Figure 6C) and podocyte loss (Figure 6D), leading to significant reductions in podocyte density (Figure 6E) and progressively exacerbated podocyte hypertrophy (Figure $6 \mathrm{~F}$ ). Importantly, podocyte number presented a strong association with PEC activation (Figure 6G).

While glomeruli from Pod-TSC $1^{+/+}$mice showed a strong association between podocyte number and glomerular volume, there was no correlation between both parameters in glomeruli from Pod-TSC1 ${ }^{-/-}$mice (Figure $6 \mathrm{H}$ ), suggesting dramatic podocyte loss across the entire glomerular size spectrum. As glomeruli underwent hypertrophy in Pod-TSC1 ${ }^{-/}$mice, podocyte hypertrophy was more pronounced (Figure 6I). Furthermore, as podocyte loss progressed in Pod-TSC1 ${ }^{-/-}$mice, podocyte hypertrophy was exacerbated (Figure $6 \mathrm{~J}$ ). Changes in glomerular size (Supplemental Figure 5C) or podocyte volume (Supplemental Figure 5D) did not correlate with PEC activation. Together, these findings indicate that exaggerated podocyte hypertrophy functions as an indirect driver of PEC activation by facilitating accelerated and progressive podocyte loss.

Pharmacological mTOR inhibition impairs mouse podocyte hypertrophy. Next, we explored the consequences of pharmacological mTOR inhibition during active podocyte loss. RAD001 (mTORC1 inhibitor) and INK128 (global mTOR inhibitor) were administered daily starting 24 hours before DT injection and throughout 3

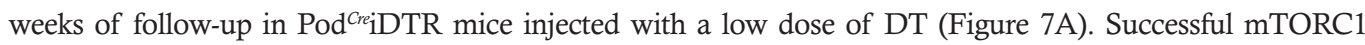
inhibition was confirmed via indirect immunofluorescence and confocal microscopy using p-rp-S6 (Figure 7B). Systemic administration of mTOR inhibitors was directly associated with exacerbated and persistent albuminuria, reaching levels observed in mice injected with high-dose DT (Figure 7C). Three weeks after DT injection, glomerulosclerotic lesions were identified only in mice with mTOR inhibition (Figure 7D) and were observed with a similar frequency as in mice with high-dose DT (Figure 7E). Pharmacological mTOR inhibition in noninjected Pod ${ }^{\mathrm{Cr}} \mathrm{iDTR}$ mice did not lead to albuminuria (Supplemental Figure 6A), podocyte loss (Supplemental Figure 6B), PEC activation (Supplemental Figure 6C), or glomerulosclerosis (Supplemental Figure 6D). In

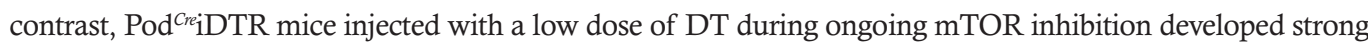
PEC activation (Figure 7F). Unlike all of our previous models, podocyte loss (Figure 7G) was not matched by compensatory podocyte hypertrophy (Figure $7 \mathrm{H})$. 
A

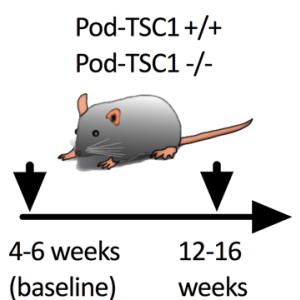

isolated podocytes
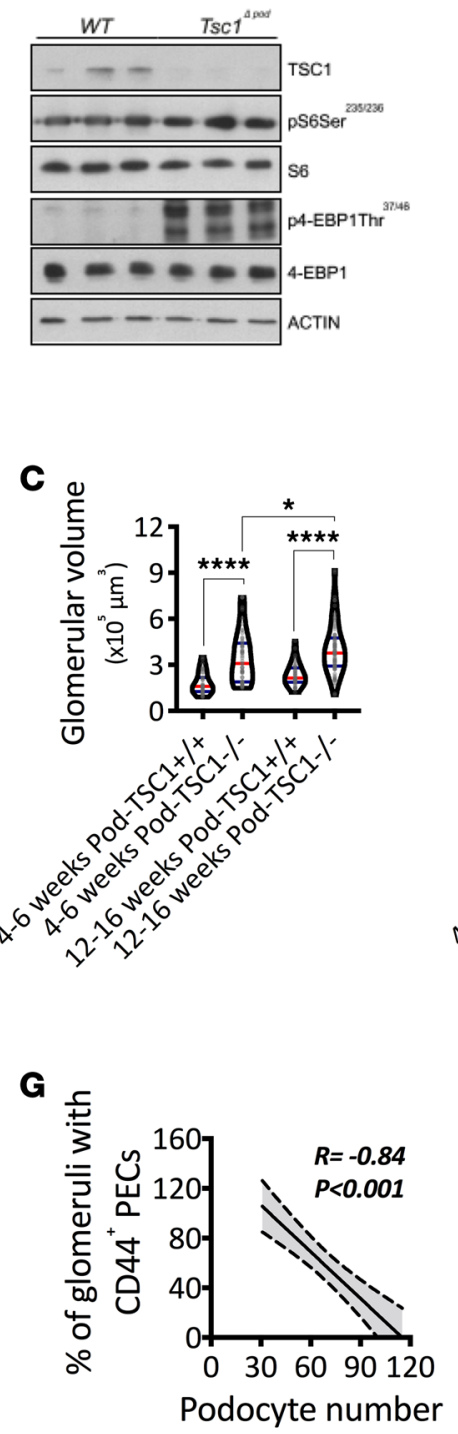


D


$\left(\times 10^{4} \mu m^{3}\right)$
E
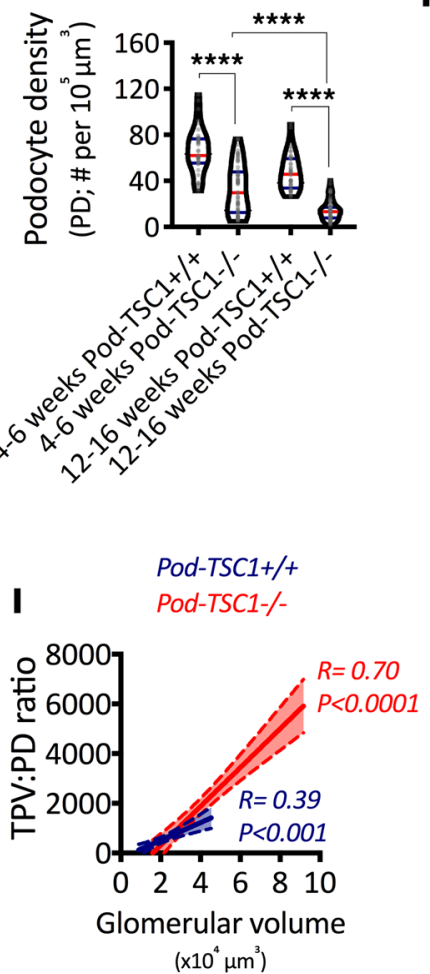

F
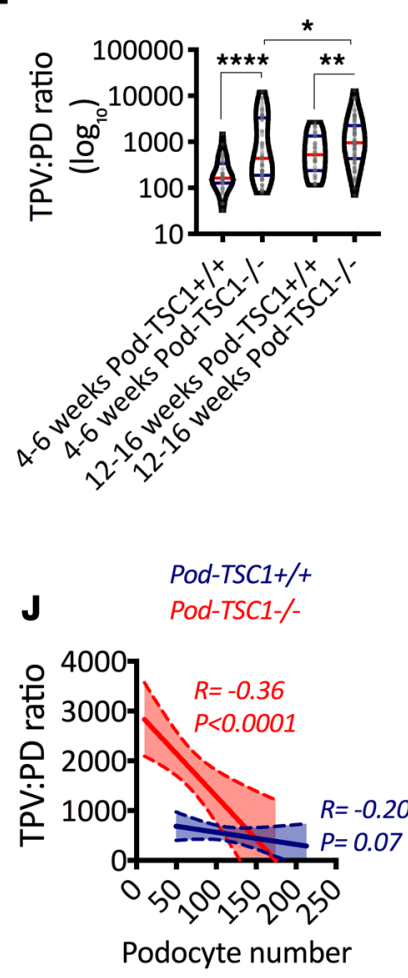

Figure 6. mTORC1 hyperactivation in podocytes leads to podocyte loss and PEC activation. (A) Schematic representation of experimental design and validation of tuberous sclerosis complex 1-KO (TSC1-KO) in isolated podocytes. (B) Representative confocal images after indirect immunofluorescence for a podocyte specific marker (Synaptopodin, SNP) and a PEC activation marker (CD44) combined with the percentage of glomeruli with CD44 ${ }^{+}$PECs. (C) Glomerular volume. (D) Podocyte number. (E) Podocyte density. (F) Total podocyte volume per unit of podocyte density (TPV:PD ratio). (C) Associations between percentage of glomeruli with $\mathrm{CD}_{4} 4^{+} \mathrm{PECs}$ and podocyte number. (H) Associations between podocyte number and glomerular volume. (I) Associations between TPV:PD and glomerular volume. (J) Associations between TPV:PD and podocyte number. ${ }^{* * *} P<0.0001 ;{ }^{* *} P<0.01 ;{ }^{*} P<0.05$. In $\mathbf{B}$, bars represent means and error bars \pm SEMs. Each dot represents 1 mouse. In violin plots, red lines represent medians and blue lines represent IQRs; every gray dot represents 1 glomerulus. Kruskal-Wallis with Dunn's multiple comparisons tests were used. In correlation analyses (Spearman's rank coefficients), solid lines represent the regression line and dotted lines the $95 \% \mathrm{Cl}$. Scale bars: $80 \mu \mathrm{m}$. 
A

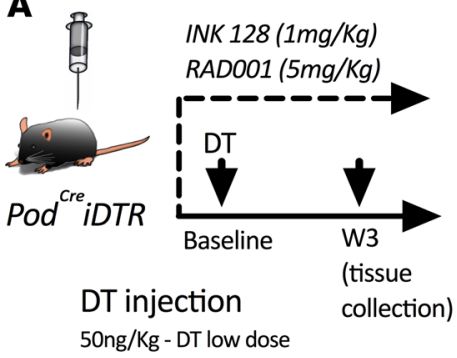

B

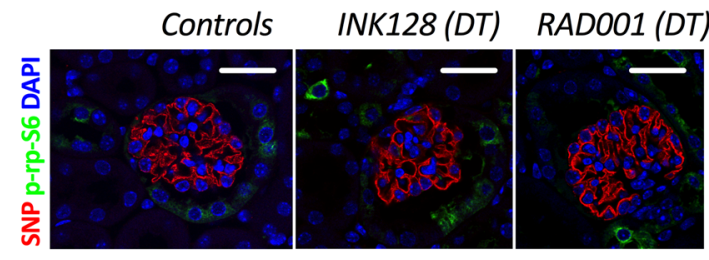

C

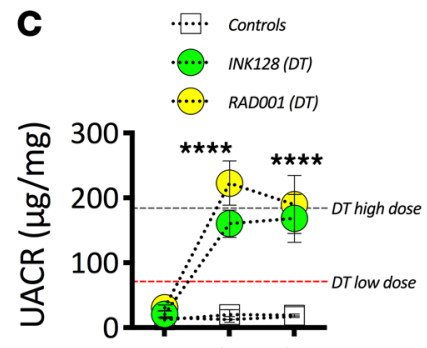

D



E

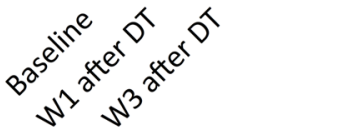

$\mathbf{F}$

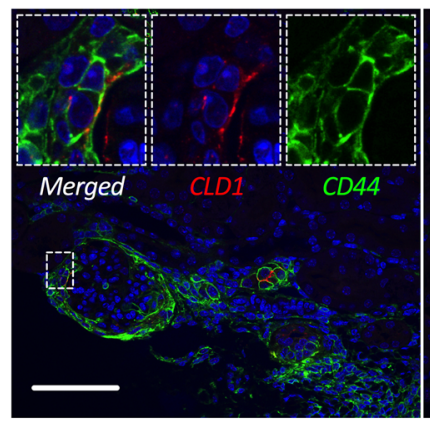

RAD001 (DT)

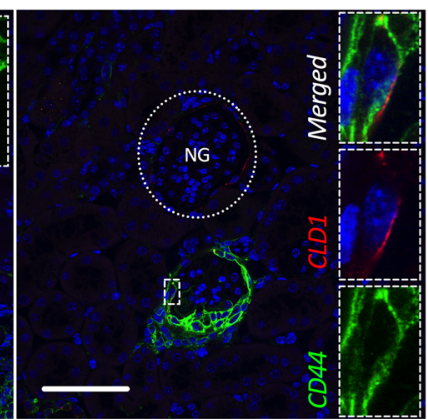

G

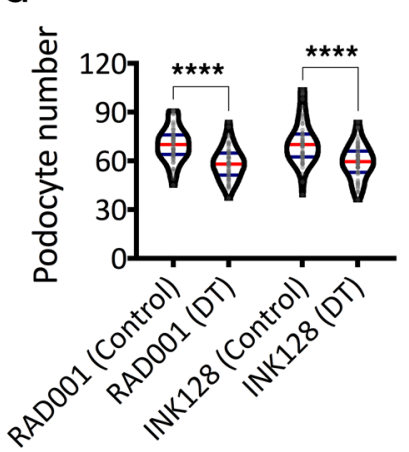

H

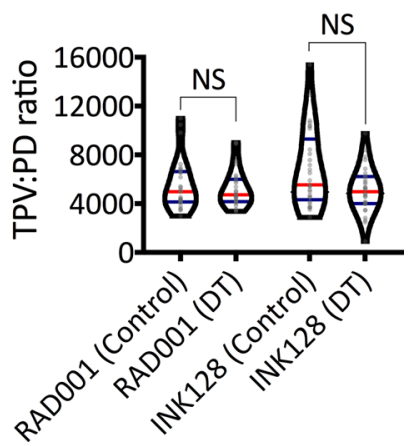

Figure 7. Pharmacological inhibition of mTOR signaling impairs podocyte hypertrophy and leads to FSCS. (A) Schematic representation of experimental design. (B) Representative confocal images after indirect immunofluorescence showing pharmacological inhibition of ribosomal protein $\mathrm{S6}$ phosphoryIation (p-rp-S6; green) in podocytes (Synaptopodin; SNP in red). (C) Urinary albumin to creatinine ratio (UACR). (D) Periodic acid-Schiff (PAS) histological stainings showing glomerulosclerosis in mTOR-treated mice during induction of podocyte loss. (E) Percentage of pathological glomeruli. (F) Representative confocal images after indirect immunofluorescence showing de novo PEC activation (CD44 upregulation; green) in mice treated with mTOR inhibitors during induction of podocyte loss via diphtheria toxin (DT) administration. (G) Podocyte number. (H) Total podocyte volume per unit of podocyte density (TPV:PD ratio). ${ }^{* * *} P<0.0001 ;{ }^{* * *} P<0.001 ;{ }^{* *} P<0.01$. In $\mathbf{C}$ and $\mathbf{E}$, circles and bars represent means and error bars \pm SEM. In violin plots, red lines represent medians and blue lines represent IQRs; every gray dot represents 1 glomerulus. Kruskal-Wallis with Dunn's multiple comparisons tests were used. Scale bars: (B) $30 \mu \mathrm{m}$, (D) $60 \mu \mathrm{m}$, and (F) $50 \mu \mathrm{m}$.

Given that pharmacological mTOR inhibition is not cell specific, we used in vitro systems in order to test the direct effect of mTOR inhibitors on podocytes and PECs. First, we induced podocyte hypertrophy via serum starvation/stimulation (Supplemental Figure 7A). We were able to clearly inhibit the hypertrophic response of podocytes in vitro using both RAD001 and INK128 (Supplemental Figure 7B). Then, we tested PEC migration as a functional readout of PEC activation in vitro. Under normal in vitro conditions, PECs are under a state of mild activation and are able to dynamically migrate. The addition of RAD001 or INK128 did not affect their migration speed (Supplemental Figure 7C) or transcriptional regulation of proactivation factors CD44 or COL4 (Supplemental Figure 7D). These results confirm a physiological role of mTOR-mediated podocyte hypertrophy in the regulation of glomerular functional integrity during periods of acute podocyte loss.

Identifying the limits of podocyte loss and hypertrophy in mice and humans. Finally, we aggregated all our morphometric data and summarized it in Figure 8. Percentages of podocyte depletion and hypertrophy 
A

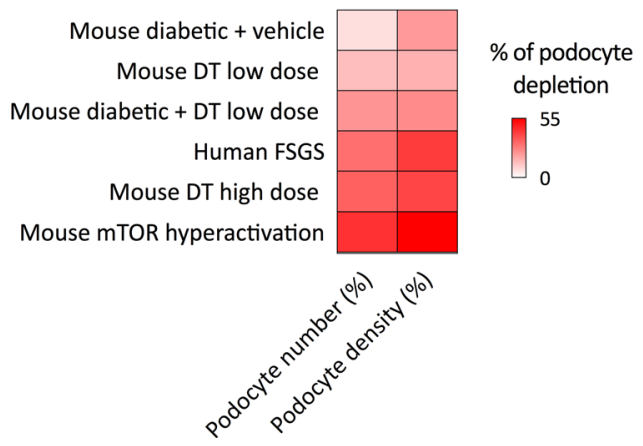

B

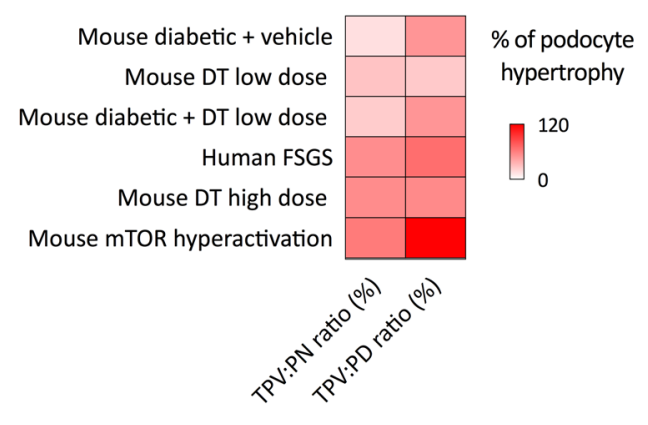

C normal PECS \& normal glomeruli
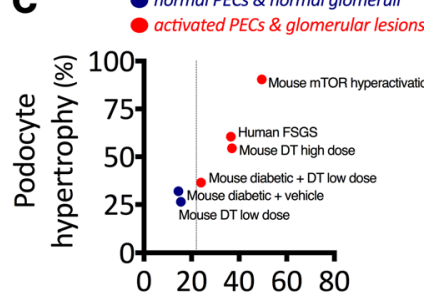

Podocyte depletion (\%)

Figure 8. The limits of podocyte loss and hypertrophy. (A and B) Respective comparisons of different indices of podocyte depletion and hypertrophy (in percent relative to controls) in each experimental setting presented in this study. (C) Association between percentage of podocyte hypertrophy and percentage of podocyte depletion. In C, each dot represents the average of 1 experiment; interrupted line shows the threshold of podocyte depletion, after which PEC activation and glomerulosclerosis are detectable.

(experimental group vs. control as normalization strategy) were calculated (Figure 8, A and B). Using both models and combined data from mice and humans, we identified that the apparent limit of podocyte depletion lies below $24 \%$ of podocyte depletion, after which PEC activation and glomerulosclerosis were consistently identified, despite dramatic increases in podocyte size (Figure 8C). The best example of this limit can be observed in our hyperglycemic mice, as injection of a small dose of DT was sufficient to achieve the depletion percentage necessary to develop glomerulosclerotic lesions.

\section{Discussion}

The mTORC1 acts as a central regulator of cell size $(17,31)$. mTOR signaling is essential during kidney development and for kidney growth during physiological body growth (18). In contrast, hyperactivity of the mTOR pathway in podocytes leads to pathological changes associated with diabetic nephropathy (19) and crescentic nephritis (20). While inhibition of mTOR signaling has proven to be an effective therapeutic approach (16), the clinical use of mTOR inhibitors has been associated with proteinuria and de novo FSGS (21-27). For this reason, the use of mTOR inhibitors in glomerular diseases remains controversial.

In a recent publication, Zschiedrich et al. (28) blocked mTORC1 signaling in podocytes and performed subtotal nephrectomy, a model of glomerular hypertrophy, which showcased the protective role of mTORC1 as a physiological response. Nishizono et al. (29) also showed that modulation of the mTORC1 pathway in podocytes impaired their capacity to adapt to exacerbated glomerular hypertrophy, thereby leading to FSGS. Our findings clearly agree with the protective role of podocyte hypertrophy proposed by both groups and provide a potentially new perspective based on the importance of mTOR-mediated podocyte hypertrophy for functional glomerular integrity following podocyte loss, independent of glomerular size. We hypothesize that patients experience cyclic episodes of podocyte loss during glomerular stress, mostly in association with known CKD risk factors such as aging, hypertension, and obesity (7). Therefore, understanding the potential consequences of an intervention that actively blocks this signaling pathway has direct clinical relevance.

Our data show that inhibition of mTOR signaling during the induction of podocyte loss leads to PEC activation and glomerulosclerosis, as the protective hypertrophic response of podocytes is significantly impaired. With this information, our current study expands on the therapeutic recommendations made by Zschiedrich et al. (28), suggesting that mTOR inhibition should be avoided in patients with immediate requirements for mTOR-mediated podocyte hypertrophy (i.e., patients with active podocyte loss). This raises an important issue: how can physicians determine if active podocyte loss is taking place in their patients? While a definitive answer is not available, an interesting alternative may be found in the analysis of urinary markers of podocyte loss (32-34). However, we believe there is a growing need for the development of noninvasive methods to assess podocyte loss.

In a recent report (35), we showed in human autopsy tissue that, even when large adult glomeruli contained more podocytes than small glomeruli from young children, the extent of glomerular hypertrophy from childhood to adulthood resulted in a marked reduction in podocyte density. Our current analyses of human tissue add to our previous findings by showing that podocyte hypertrophy is present in subjects 
without kidney disease during normal somatic growth, which in turn drives glomerular hypertrophy to compensate for increasing metabolic demands in adulthood $(36,37)$, as previously described by Kikuchi et al. (38) using different quantification approaches and experimental designs. For these reasons, the role of mTOR-mediated podocyte hypertrophy should be carefully examined in periods of rapid body growth (puberty, pregnancy) or expected compensatory glomerular hypertrophy/hyperfiltration.

Our current data also show that reductions in podocyte density and increases in podocyte size are both exacerbated in human FSGS, suggesting that podocyte hypertrophy was not able to match the loss of podocytes and preserve glomerular integrity. Previous work by Hodgin et al. (39) showed that age-related reductions in podocyte density lead to podocyte hypertrophy, which may place podocytes under stress and eventually facilitate the development of glomerulosclerosis. Furthermore, Fukuda et al. (40) reported that angiotensin-2mediated progressive podocyte loss led to destabilization of glomeruli with severe podocyte depletion. Both studies elegantly highlight that podocyte hypertrophy has a limit when subjected to chronic or significant stress. We explored the limits of podocyte hypertrophy using 2 different approaches: (a) a 1-hit model as we induced selective podocyte loss beyond the compensatory limit of podocyte hypertrophy, and (b) a 2-hit model as we generated an adverse environment (i.e., hyperglycemia) and induced subsequent selective podocyte loss that, under normal conditions, is tolerated. The results in both cases were identical, demonstrating exacerbated podocyte hypertrophy combined with PEC activation and glomerulosclerosis, raising an important question: why is podocyte hypertrophy unable to preserve functional glomerular integrity in such settings?

Unfortunately, the answer to this question lies beyond the scope of our study. It has been proposed that podocyte loss leaves denuded areas of glomerular basement membrane, allowing molecules that would normally mainly remain in capillaries to more readily cross into the urinary space, including albumin, and growth factors (such as PDGF). Hakroush et al. (41) has shown that severe podocyte depletion leads to rapid PEC activation and glomerulosclerotic lesions. We postulate that PECs are able to quickly identify the "leakiness" of the glomerular filtration barrier $(42,43)$, which will serve as a trigger for PEC activation. Interestingly, a recent publication shows that blocking tetraspanin CD9 signaling in PECs is sufficient to prevent glomerulosclerosis (44), which may be directly related to the capacity of PECs to sense signals from neighboring cells (e.g., endothelial cells or podocytes). Thus, there is growing interest in the field of PEC biology as they emerge as potential therapeutic targets.

PECs have also gained attention as potential sources of additional podocytes in the postnatal period (14). Recent publications have shown that podocyte regeneration may be possible in specific scenarios (45-51), but other publications have ruled this out $(10,52)$. Conflicting evidence may highlight differences in glomerular injury models, mouse backgrounds, adequacy of wash-out periods, transgene leakiness, and quantification methods, among other factors. In the current study, we have performed multiple experiments where the main compensatory response is podocyte hypertrophy in the absence of detectable podocyte regeneration. While it is possible that small (negligible) postnatal podocyte gain may be missed in our studies, podocyte hypertrophy appears to be a much more efficient compensatory response in order to cope with podocyte depletion.

It is also worth noting that podocyte hypertrophy has a physiological limit. Wiggins et al. (53) proposed stages for podocyte hypertrophy and suggested that there is a limit for podocyte growth that, when reached, would lead to podocyte detachment, creating a vicious cycle of podocyte loss and glomerulosclerosis. Building on this concept, Zschiedrich et al. (28) proposed a therapeutic window for mTOR inhibition, as the compensatory function of podocyte hypertrophy is exhausted and exacerbated podocyte hypertrophy compromises podocyte integrity, leading to podocyte loss. Our findings in Pod-TSC1 ${ }^{-/-}$mice are in agreement with both of these studies, as we quantified excessive levels of podocyte hypertrophy induced by hyperactivation of mTORC1. Importantly, the development of FSGS in Pod-TSC1 ${ }^{-1}$ mice directly correlated with glomerular hypertrophy and accelerated podocyte loss, leading to PEC activation. Together, mTORC1 hyperactivation in podocytes remains an attractive therapeutic target. We propose that future studies should carefully determine surrogate markers for a safe therapeutic window, aiming to control exaggerated podocyte hypertrophy without interfering with its compensatory role.

In conclusion, this study shows a critical protective role of mTOR-mediated podocyte hypertrophy after podocyte loss in the absence of detectable podocyte regeneration. Our findings suggest that podocyte hypertrophy regulates glomerular integrity until a threshold of podocyte depletion is reached, after which even exacerbated podocyte hypertrophy is unable to prevent PEC activation and glomerulosclerosis. Furthermore, excessive mTOR-mediated podocyte hypertrophy enhances podocyte loss and perpetuates a cycle of podocyte depletion and glomerulosclerosis. 


\section{Methods}

Human tissue. Tissue collection from autopsies was performed at the University of Mississippi Medical Center. After fixation with $10 \%$ formalin, representative kidney blocks from the upper pole and mid portion of the kidney were embedded in paraffin, as previously described (54). Adult control tissue was obtained from nephrectomies due to renal cell carcinomas, and biopsy samples from FSGS patients were obtained from routine clinical investigations performed at Monash Health (Melbourne, Australia; ethics approval: 09092B). Additional biopsies were collected at Hamburg-Eppendorf Medical Centre after informed consent from each patient in accordance to the Declaration of Helsinki. Tissue was used for validation proposes and underwent careful double anonymization.

$m R N A$ analysis of human biopsies. Human renal biopsy specimens and Affymetrix microarray expression data were obtained within the framework of the European Renal cDNA Bank's Kröner-Fresenius Biopsy Bank (55). Biopsies were obtained from patients after informed consent and with approval of the local ethics committees. Following renal biopsy, the tissue was transferred to RNase inhibitor and micro-dissected into glomeruli and tubulo-interstitium. Total RNA was isolated from micro-dissected glomeruli, reverse transcribed, and linearly amplified according to a protocol previously reported (56).

Affymetrix GeneChip Human Genome U133A and U133 Plus2.0 Arrays were used in this study. The published microarray expression data used in this study came from individual patients with diabetic nephropathy (DN, $n=14$ ) and FSGS $(n=23)$. Pretransplantation kidney biopsies from living donors (LD, $n=42$ ) were used as control renal tissue (Gene Expression Omnibus Database accession numbers GSE99340, GSE32591, GSE35489, GSE37463). Fragmentation, hybridization, staining, and imaging were performed according to the Affymetrix Expression Analysis Technical Manual (Affymetrix). Raw data was normalized using Robust Multichip Algorithm (RMA) and annotated by Human Entrez Gene custom CDF annotation version 18 (http://brainarray.mbni.med.umich.edu/Brainarray/Database/ CustomCDF/genomic_curated_CDF.asp).

The log-transformed dataset was corrected for batch effect using ComBat from the GenePattern pipeline (http://www.broadinstitute.org/cancer/software/genepattern). To identify differentially expressed genes, the Significance analysis of Microarrays (SAM) method was applied using TiGR (MeV, Version 4.8.1) (57). A $q$ value below $5 \%$ was considered to be statistically significant.

Pod ${ }^{\text {Cri } i D T R, ~ P o d-r t T A-m T / m G-i D T R, ~ a n d ~ P o d-T S C 1-1-~ m o u s e ~ m o d e l s . ~ A l l ~ a n i m a l ~ e x p e r i m e n t s ~ w e r e ~ c o n d u c t e d ~}$ in accordance with guidelines provided by the Monash University Animal Research Platform(MARP/2014/015) and by the Committee on Research Animal Care, Regierungspräsidium Freiburg (Pod-rtTA-mT/mG-iDTR, and Pod-TSC1 ${ }^{-/}$mice). The iDTR mouse line (CBy.B6Gt[ROSA]26Sortm1[HBEGF]Awai/J) used in the generation of Pod-rtTA-mT/mG-iDTR mice was a gift of Ari Waisman (Johannes-Gutenberg University, Mainz, Germany) (58). Pod ${ }^{C r i D T R}$ mice were used for all other experiments as per our previous work (11). For the induction of $\mathrm{mT}$ deletion, mice received doxycycline hydrochloride (MilliporeSigma) via the drinking water (2 $\mathrm{mg} / \mathrm{ml}$ with $5 \%$ sucrose) for 72 hours at 4 weeks of age. After a washout period of one week, DT (MilliporeSigma) was dissolved in PBS and injected i.p. at $0(n=11), 2(n=2), 3(n=12)$, or $5(n=7)$ ng DT/kg of BW. These doses were optimized for each mouse strain. Thus, we decided to classify mice based on their response to DT injection and the development of glomerulosclerosis rather than the dose of DT. Importantly, in both experiments, the results were identical, so we believe that the different responses to DT rely on the differences in mouse backgrounds/strains.

Hyperglycemia. Hyperglycemia was induced via 5 consecutive i.p. injections of STZ (Abcam, ab142155) diluted in $10 \mathrm{mM}$ citrate buffer ( $\mathrm{pH} 4.6)$ at a dose of $55 \mathrm{mg} / \mathrm{kg}$ (100 $\mu \mathrm{l}$ bolus). Fasting blood glucose (5 hours) was monitored at baseline (before the first injection), 9 days after the last injection (to confirm hyperglycemia), and then weekly until the experiment was finalized. Measurements were performed at the same time of day to minimize any effects of circadian rhythm.

$U A C R$. Spot urine samples were collected during 3 different phases after i.p. injection of DT: baseline (0-3 days), acute (4-9 days), and chronic (10+ days). Samples were stored at $-80^{\circ} \mathrm{C}$ and then used for the estimation of a UACR. The Albuwell murine microalbuminuria ELISA (Exocell) and Microflural Microalbumin Test kit (Progen) were used to determine the albumin concentration in urine. The creatinine companion (Exocell), a Creatinine PAP kit (Labortechnik) and a micro-plate assay were used to determine urinary creatinine concentration.

Assessment of podocyte regeneration based on flow cytometry. Glomeruli were isolated by Dynabead perfusion as described previously (59). After digestion to single cells, the cells were fixed in 4\% PFA for 3 minutes, permeabilized with $0.5 \%$ Triton for 12 minutes, and stained with anti-podocin antibody (MilliporeSigma, P0372), 
labeled with Alexa Fluor 647 (Invitrogen, Zenon labeling kit) for 1 hour. Stained glomerular cells were resuspended in FACS buffer (0.5\% BSA, 5 mM EDTA in PBS) and analyzed using FACS Calibur (BD Biosciences).

mTOR pharmacological inhibition. mTOR inhibitors were administered by daily oral gavage starting 48 hours prior to DT injection until the endpoint was reached. RAD001 (Selleckchem, S1120) was administered at a dose of $5 \mathrm{mg} / \mathrm{kg}$ at a concentration of $6 \mathrm{mg} / \mathrm{ml}$ in $50 \mathrm{mM}$ sodium phosphate plus $1 \%$ methylcellulose (M7027-100G; Sigma-Aldrich). INK128 (Selleckchem, S2811) was administered at a dose of $1 \mathrm{mg} / \mathrm{kg}$ at a concentration of $1.5 \mathrm{mg} / \mathrm{ml}$ in pure ethanol further diluted to a concentration of $0.3 \mathrm{mg} / \mathrm{ml}$ in $50 \mathrm{mM}$ sodium phosphate plus $1 \%$ methyl cellulose.

Immunofluorescence. We applied previously reported protocols for $4-\mu \mathrm{m}$ paraffin sections (35) and for $800-\mu \mathrm{m}$ slices (11). A list of all used antibodies is provided in Supplemental Table 4.

Confocal microscopy of thin sections. Optical images were obtained using inverted laser confocal microscopes (SP5, Leica Microsystems; LSM800, Zeiss). Representative images were obtained with 8 line averages and stored in $1024 \times 1024$ - or $2048 \times 2048$-pixel frames, depending on the experiment and microscopy setting.

Optical clearing and confocal microscopy. Optical clearing was performed via solvent-based clearing (Benzyl Alcohol, Benzyl Benzoate; BABB) as previously described (11). A Leica SP8 Multiphoton Microscope (Leica Microsystems) fitted with a $20 \times$ BABB objective lens (0.95 numerical aperture; $1950 \mu$ m working distance) was used. Serial optical images were obtained at $1-\mu \mathrm{m}$ intervals and stored in $1024 \times 1024$-pixel frames. This approach allowed us to estimate podocyte depletion parameters in whole glomeruli.

Model-based stereology for human and mouse podometrics. Model-based stereology (60) was applied to confocal images and allowed the estimation of podocyte number per glomerulus, podocyte density, and average podocyte volume in human samples. All glomeruli were analyzed, including sclerotic and nonsclerotic in cases of FSGS. In segmental lesions, glomerular area was delineated as the entire glomerular tuft. Fiji imaging software (Max Planck Institute of Molecular Cell Biology and Genetics, Dresden, Germany) was used to navigate the raw files. Podocytes were defined as DAPI ${ }^{+} \mathrm{WT}-1^{+}, \mathrm{DAPI}^{+} \mathrm{p} 57^{+}$, or $\mathrm{DAPI}^{+} \mathrm{SNP}^{+}$ cells, depending on the experiment. The cell counter built-in feature (http://rsbweb.nih.gov/ij/plugins/ cell-counter.html) was used to keep track of podocyte nuclear counts. Podocyte nuclear and cytoplasmic areas were obtained in order to calculate podocyte number, density, and volume; glomerular cross-sectional area was measured in order to estimate glomerular volume and, thereby, define podocyte density.

Whole glomerular analysis for 3-D podometrics in mice. Fiji imaging software (61) was used to move through the $z$ axis of each stack of optical sections. Podocytes were defined as $\mathrm{p} 57^{+} \mathrm{SNP}^{+}$cells. The cell counter built-in feature (http://rsbweb.nih.gov/ij/plugins/cell-counter.html) was used to keep track of podocyte nuclear counts while moving through the entire glomerulus (from top to bottom). Estimation of glomerular and podocyte volume was performed using 3-D rendering and analysis software (Imaris, version 8; Bitplane AG) as previously reported (11).

In vitro regulation of podocyte cytoskeleton. Primary podocytes of proven origin (62) were serum starved for 24 hours and then stimulated with 10\% and 20\% serum. Podocyte nuclear and cytoplasmic areas were measured by Phalloidin fluorescent staining by ActinRed reagent (Invitrogen, R37112) with a nuclear counterstain (Hoechst 33342; MilliporeSigma). Confocal images were obtained with a confocal laser microscope (LSM 710; Zeiss) running the Zen 2012 (Zeiss) software.

In vitro analysis of cultured PECS. Primary PECs of proven origin (62) were used for both migration essays and RNA analyses. For migration essays, PECs were exposed to doses of $500 \mathrm{ng} / \mathrm{ml}$ of both INK128 (Selleckchem, S2811) and RAD001 (Selleckchem, S1120) in $\mu$-Dish 35 mm (IBIDI, 81176) - images were taken at 4 and 8 hours after drug administration. For RNA analyses, PECs were exposed to both drugs at a concentration of $100 \mathrm{nM}$ for 48 hours before RNA isolation. Total RNA was isolated using the Invisorb Spin CellRNA Mini Kit (Invitek). The RNA content and sample purity were determined using a NanoDrop (Thermo Fisher Scientific). The cDNA synthesis were performed in a $30-\mu 1$ reaction mix including $1 \mu \mathrm{g}$ of total RNA, $1 \mu \mathrm{l}$ of random primer (6 nucleotides in length, $250 \mathrm{ng} / \mu \mathrm{l}$, Roche Diagnostics), $6 \mu 1$ of M-MLV reverse transcriptase buffer (Invitrogen), $1.5 \mu 1$ of dNTP-mix (10 mM each, Roth), $0.7 \mu 1$ of RNase-inhibitor (40 U/ $\mu 1$, Promega), $1 \mu \mathrm{l}$ of M-MLV reverse transcriptase (200 U/ $\mu 1$; Invitrogen), and RNAse-free water. The mix was incubated for 10 minutes at $25^{\circ} \mathrm{C}$, followed by 1 hour at $42^{\circ} \mathrm{C}$. Quantitative PCR (qPCR) was carried out using an ABI Prism 7300 sequence detector (Applied Biosystems). In each reaction, $0.75 \mu 1$ of cDNA and the qPCR Core Kit or the qPCR Core Kit of SyBr Green I (both Eurogentec) were used in a total of $25 \mu 1$ volume. The PCR conditions were $50^{\circ} \mathrm{C}$ for 2 minutes; $95^{\circ} \mathrm{C}$ for 10 minutes, followed by 40 cycles of $95^{\circ} \mathrm{C}$ for 15 seconds; and $60^{\circ} \mathrm{C}$ for 1 minute. Primers and probes were designed from sequences in the GenBank database using the Primer Express software (Applied Biosystems). All quantitative data from the real-time 
RT-PCR were normalized using GAPDH as an internal control and calculated using the $\Delta \Delta$ Ct-method. Primers included: CD44 (forward: 5' - TCC GAA TTA GCT GGA CAC TC - 3'; and reverse: 5' - CCA CAC CTT CTC CTA CTA TTG AC - 3'), SSECKs (forward: 5' - GCA CAC TCC AAA CTT TCA TCC AT - 3'; and reverse: 5' - AGA TTG CAT AGA GGT TCA AGT TCA AA - 3'), COL4 (forward: 5' - GGC GGT ACA CAG TCA GAC CAT - 3'; reverse: 5' - TGG TGT GCA TCA CGA AGG A - 3'; and probe: 5' - TCC GCA GTG CCC TAA CGG TTG GT - 3'), and GAPDH (forward: 5' - AGA TGG TGA TGG GCT TCC C - 3'; reverse: 5' - GGC AAA TTC AAC GGC ACA GT - 3'; and probe: 5' - AAG GCC GAG AAT GGG AAG CTT GTC ATC - 3').

Statistics. All statistical analyses were performed using GraphPad Prism (v8.0.2) and SPSS 24.0 (IBM). Results are reported as mean \pm SEM or median and interquartile range (IQR) depending on normality test - most of the morphometric analyses were reported as violin plots showing median, IQR, and data distribution. Significance was evaluated using the unpaired Mann Whitney's $U$ test when comparing 2 continuous variables. For comparison of 3 groups, Kruskal-Wallis test with Dunn's multiple comparisons test was used. Correlation analyses were performed using Spearman's correlations. Bootstrapping was applied to obtain 1000 times resampling and derive the corresponding 95\% CI for the correlation coefficient. A $P$ value or $q$ value below 0.05 was considered to be statistically significant.

Study approval. Ethics approval was obtained in advance from the Institutional Review Board of the University of Mississippi Medical Center and the Monash University Human Research Ethics Committee (2006/753). Biopsy samples from FSGS patients were obtained from routine clinical investigations performed at Monash Health (Melbourne, Australia; ethics approval: 09092B). Additional biopsies were collected at Hamburg-Eppendorf Medical Centre after informed consent from each patient in accordance to the Declaration of Helsinki. All animal experiments were conducted in accordance with guidelines provided by the Monash University Animal Research Platform (MARP/2014/015) and by the Committee on Research Animal Care, Regierungspräsidium Freiburg (Pod-rtTA-mT/mG-iDTR, and Pod-TSC1 ${ }^{-/-}$mice).

\section{Author contributions}

All authors revised the manuscript before submission. VGP, JWVDW, NW, LACM, TB, MTL, LG, MNW, FB, MS, CDC, CK, RK, TS, CVR, LT, RR, and YBYS assisted with experiments and data analyses. MMK, JL, GMN, KMD, LF, and MJM provided experimental resources and expertise. MDH, WEH, SDR, PGK, FP, and TW were responsible for collection of human samples. VGP, TBH, DJNP, and JFB were responsible for the study design, interpretation of data, writing of the manuscript, and study supervision.

\section{Acknowledgments}

This research was supported by multiple funding agencies, including: National Health and Medical research Council (NHMRC) of Australia to VGP (CJ Martin Research Fellowship; 1128582) and to JFB, DNP, KMD, LF, and WEH (1141339, 1065902, 1041844, and 1121793); Department of Health and Human Services acting through the Victorian Cancer Agency (MCRF16007) and Cancer Australia (CA1084546) to LF; the Colonial Foundation (2001-2011) to WEH; Department of Foreign Affairs and Trade of Australia to WEH (Australia Fellowship; 511081); the Humboldt Foundation and German Society of Nephrology (DGfN) to VGP; the European Research Council (677448) to RF and (616891) to TBH; German Research Foundation to RK (KR-4073/3-1, SCHN1188/5-1, CRC/TRR57), to TW (CRC/1192), to TS (332853055), to MJM (Heisenberg Fellowship; MO1082/7-1), and to TBH (CRC/1192, CRC/1140, CRC/992, HU 1016/82, and Heisenberg Fellowship); the German Ministry for Science and Education (BMBF "STOP-FSGS", 01GM1518A and 01GM1518C to MJM and TBH, respectively); the Excellence Initiative of the German Federal and State Governments (BIOSS); the Freiburg Institute for Advanced Studies; Else Kröner-Fresenius-Foundation programs NAKSYS and iPRIME to TBH; RWTH Aachen University START Grant to RK (101/15) and to CK (105/17); and the Else Kröner Fresenius Foundation to the ERCB-KFB, CK (A200/2013), and to TS (A197/2015). TBH and this project have received funding from the Innovative Medicines Initiative 2 Joint Undertaking under grant agreement no. 115974 (BEAt-DKD). This Joint Undertaking receives support from the European Union's Horizon 2020 research and innovation programme and EFPIA. This work was also supported by staff from Monash Micro Imaging and Monash Histology Platform at Monash University, Australia, and by the Immunohistochemistry and Confocal Microscopy Unit, a core facility of the Interdisciplinary Center for Clinical Research (IZKF) Aachen within the Faculty of Medicine at the RWTH Aachen University (Aachen, Germany). We also thank all participating centres of 
the European Renal cDNA Bank's Kröner-Fresenius biopsy bank (ERCB-KFB) and their patients for their cooperation. For active members at the time of the study, see ref. 63. Finally, we would like to thank the technical support of Regina Lanzmich.

Address correspondence to: Victor G. Puelles, III. Department of Medicine, University Medical Center Hamburg-Eppendorf, Martinistraße 52, Gebäude N27, 20246 Hamburg, Germany. Phone: 49.040.741035337; Email: victor.puellesrodriguez@monash.edu. Or to: John F. Bertram, Monash University, 19 Innovation Walk, 3800 Victoria, Australia. Telephone: 61.3.9902.9101; Email: john.bertram@ monash.edu. Or to: David Nikolic-Paterson, Monash Medical Centre, 246 Clayton Road, 3168 Victoria, Australia. Phone: 61.3.9594.3535; Email: david.nikolic-paterson@monash.edu. Or to: Tobias B. Huber, III. Department of Medicine, University Medical Center Hamburg-Eppendorf, Martinistraße 52, Gebäude O10, 20246 Hamburg, Germany. Phone: 49.040.7410.53908; Email: t.huber@uke.de.

1. Hill NR, et al. Global Prevalence of Chronic Kidney Disease - A Systematic Review and Meta-Analysis. PLoS ONE 2016;11(7):e0158765.

2. Eckardt KU, et al. Evolving importance of kidney disease: from subspecialty to global health burden. Lancet. 2013;382(9887):158-169.

3. Wiggins RC. The spectrum of podocytopathies: a unifying view of glomerular diseases. Kidney Int. 2007;71(12):1205-1214.

4. Assady S, Wanner N, Skorecki KL, Huber TB. New Insights into Podocyte Biology in Glomerular Health and Disease. $J$ Am Soc Nephrol. 2017;28(6):1707-1715.

5. Mulay SR, et al. Podocyte loss involves MDM2-driven mitotic catastrophe. J Pathol. 2013;230(3):322-335

6. Wharram BL, et al. Podocyte depletion causes glomerulosclerosis: diphtheria toxin-induced podocyte depletion in rats expressing human diphtheria toxin receptor transgene. J Am Soc Nephrol. 2005;16(10):2941-2952.

7. Puelles VG, et al. Human podocyte depletion in association with older age and hypertension. Am J Physiol Renal Physiol. 2016;310(7):F656-F668.

8. Weil EJ, et al. Podocyte detachment and reduced glomerular capillary endothelial fenestration promote kidney disease in type 2 diabetic nephropathy. Kidney Int. 2012;82(9):1010-1017.

9. Pagtalunan ME, et al. Podocyte loss and progressive glomerular injury in type II diabetes. J Clin Invest. 1997;99(2):342-348.

10. Wanner N, et al. Unraveling the role of podocyte turnover in glomerular aging and injury. J Am Soc Nephrol. 2014;25(4):707-716.

11. Puelles VG, et al. Validation of a Three-Dimensional Method for Counting and Sizing Podocytes in Whole Glomeruli. $J$ Am Soc Nephrol. 2016;27(10):3093-3104.

12. Moeller MJ, Kuppe C. Glomerular disease: the role of parietal epithelial cells in hyperplastic lesions. Nat Rev Nephrol. 2014;10(1):5-6.

13. Berger K, Moeller MJ. Podocytopenia, parietal epithelial cells and glomerulosclerosis. Nephrol Dial Transplant. 2014;29(5):948-950.

14. Shankland SJ, Smeets B, Pippin JW, Moeller MJ. The emergence of the glomerular parietal epithelial cell. Nat Rev Nephrol. 2014;10(3):158-173.

15. Kriz W. Glomerular diseases: podocyte hypertrophy mismatch and glomerular disease. Nat Rev Nephrol. 2012;8(11):618-619.

16. Fantus D, Rogers NM, Grahammer F, Huber TB, Thomson AW. Roles of mTOR complexes in the kidney: implications for renal disease and transplantation. Nat Rev Nephrol. 2016;12(10):587-609.

17. Laplante M, Sabatini DM. mTOR signaling in growth control and disease. Cell. 2012;149(2):274-293.

18. Gödel M, et al. Role of mTOR in podocyte function and diabetic nephropathy in humans and mice. J Clin Invest. 2011;121(6):2197-2209.

19. Inoki $\mathrm{K}$, et al. mTORC1 activation in podocytes is a critical step in the development of diabetic nephropathy in mice. $J$ Clin Invest. 2011;121(6):2181-2196.

20. Kurayama R, et al. Role of amino acid transporter LAT2 in the activation of mTORC1 pathway and the pathogenesis of crescentic glomerulonephritis. Lab Invest. 2011;91(7):992-1006.

21. Dittrich E, Schmaldienst S, Soleiman A, Hörl WH, Pohanka E. Rapamycin-associated post-transplantation glomerulonephritis and its remission after reintroduction of calcineurin-inhibitor therapy. Transpl Int. 2004;17(4):215-220.

22. Butani L. Investigation of pediatric renal transplant recipients with heavy proteinuria after sirolimus rescue. Transplantation. 2004;78(9):1362-1366

23. Izzedine H, Brocheriou I, Frances C. Post-transplantation proteinuria and sirolimus. N Engl J Med. 2005;353(19):2088-2089.

24. Sennesael JJ, Bosmans JL, Bogers JP, Verbeelen D, Verpooten GA. Conversion from cyclosporine to sirolimus in stable renal transplant recipients. Transplantation. 2005;80(11):1578-1585.

25. Letavernier E, et al. High sirolimus levels may induce focal segmental glomerulosclerosis de novo. Clin J Am Soc Nephrol. 2007;2(2):326-333.

26. Cho ME, Hurley JK, Kopp JB. Sirolimus therapy of focal segmental glomerulosclerosis is associated with nephrotoxicity. Am J Kidney Dis. 2007;49(2):310-317.

27. Hochegger K, et al. Differential effects of rapamycin in anti-GBM glomerulonephritis. J Am Soc Nephrol. 2008;19(8):1520-1529.

28. Zschiedrich S, et al. Targeting mTOR Signaling Can Prevent the Progression of FSGS. J Am Soc Nephrol. 2017;28(7):2144-2157.

29. Nishizono R, et al. FSGS as an Adaptive Response to Growth-Induced Podocyte Stress. J Am Soc Nephrol. 2017;28(10):2931-2945.

30. Lin X, Suh JH, Go G, Miner JH. Feasibility of repairing glomerular basement membrane defects in Alport syndrome. $J A m$ Soc Nephrol. 2014;25(4):687-692.

31. Saxton RA, Sabatini DM. mTOR Signaling in Growth, Metabolism, and Disease. Cell. 2017;169(2):361-371. 
32. Perez-Hernandez J, Olivares MD, Forner MJ, Chaves FJ, Cortes R, Redon J. Urinary dedifferentiated podocytes as a non-invasive biomarker of lupus nephritis. Nephrol Dial Transplant. 2016;31(5):780-789.

33. Wang P, Li M, Liu Q, Chen B, Ji Z. Detection of urinary podocytes and nephrin as markers for children with glomerular diseases. Exp Biol Med (Maywood). 2015;240(2):169-174.

34. Vogelmann SU, Nelson WJ, Myers BD, Lemley KV. Urinary excretion of viable podocytes in health and renal disease. Am J Physiol Renal Physiol. 2003;285(1):F40-F48

35. Puelles VG, et al. Podocyte Number in Children and Adults: Associations with Glomerular Size and Numbers of Other Glomerular Resident Cells. J Am Soc Nephrol. 2015;26(9):2277-2288.

36. Puelles VG, Hoy WE, Hughson MD, Diouf B, Douglas-Denton RN, Bertram JF. Glomerular number and size variability and risk for kidney disease. Curr Opin Nephrol Hypertens. 2011;20(1):7-15.

37. Puelles VG, et al. Glomerular hypertrophy in subjects with low nephron number: contributions of sex, body size and race. Nephrol Dial Transplant. 2014;29(9):1686-1695.

38. Kikuchi M, Wickman L, Rabah R, Wiggins RC. Podocyte number and density changes during early human life. Pediatr Nephrol. 2017;32(5):823-834

39. Hodgin JB, et al. Glomerular Aging and Focal Global Glomerulosclerosis: A Podometric Perspective. J Am Soc Nephrol. 2015;26(12):3162-3178

40. Fukuda A, et al. Angiotensin II-dependent persistent podocyte loss from destabilized glomeruli causes progression of end stage kidney disease. Kidney Int. 2012;81(1):40-55.

41. Hakroush S, Cebulla A, Schaldecker T, Behr D, Mundel P, Weins A. Extensive podocyte loss triggers a rapid parietal epithelial cell response. J Am Soc Nephrol. 2014;25(5):927-938.

42. Alpers CE, Seifert RA, Hudkins KL, Johnson RJ, Bowen-Pope DF. PDGF-receptor localizes to mesangial, parietal epithelial, and interstitial cells in human and primate kidneys. Kidney Int. 1993;43(2):286-294.

43. Moeller MJ, Smeets B. Novel target in the treatment of RPGN: the activated parietal cell. Nephrol Dial Transplant. 2013;28(3):489-492.

44. Lazareth $\mathrm{H}$, et al. The tetraspanin CD9 controls migration and proliferation of parietal epithelial cells and glomerular disease progression. Nat Commun. 2019;10(1):3303.

45. Eng DG, Sunseri MW, Kaverina NV, Roeder SS, Pippin JW, Shankland SJ. Glomerular parietal epithelial cells contribute to adult podocyte regeneration in experimental focal segmental glomerulosclerosis. Kidney Int. 2015;88(5):999-1012.

46. Kaverina NV, Eng DG, Schneider RR, Pippin JW, Shankland SJ. Partial podocyte replenishment in experimental FSGS derives from nonpodocyte sources. Am J Physiol Renal Physiol. 2016;310(11):F1397-F1413.

47. Kaverina NV, et al. WT1 Is Necessary for the Proliferation and Migration of Cells of Renin Lineage Following Kidney Podocyte Depletion. Stem Cell Reports. 2017;9(4):1152-1166.

48. Kaverina NV, et al. Tracking the stochastic fate of cells of the renin lineage after podocyte depletion using multicolor reporters and intravital imaging. PLOS ONE. 2017;12(3):e0173891.

49. Eng DG, et al. Detection of renin lineage cell transdifferentiation to podocytes in the kidney glomerulus with dual lineage tracing. Kidney Int. 2018;93(5):1240-1246.

50. Romoli S, et al. CXCL12 blockade preferentially regenerates lost podocytes in cortical nephrons by targeting an intrinsic podocyte-progenitor feedback mechanism. Kidney Int. 2018;94(6):1111-1126.

51. Lasagni L, et al. Podocyte Regeneration Driven by Renal Progenitors Determines Glomerular Disease Remission and Can Be Pharmacologically Enhanced. Stem Cell Reports. 2015;5(2):248-263.

52. Berger K, et al. The regenerative potential of parietal epithelial cells in adult mice. J Am Soc Nephrol. 2014;25(4):693-705

53. Wiggins JE, et al. Podocyte hypertrophy, "adaptation," and "decompensation" associated with glomerular enlargement and glomerulosclerosis in the aging rat: prevention by calorie restriction. J Am Soc Nephrol. 2005;16(10):2953-2966.

54. Hughson M, Farris AB, Douglas-Denton R, Hoy WE, Bertram JF. Glomerular number and size in autopsy kidneys: the relationship to birth weight. Kidney Int. 2003;63(6):2113-2122.

55. Cohen CD, Frach K, Schlöndorff D, Kretzler M. Quantitative gene expression analysis in renal biopsies: a novel protocol for a high-throughput multicenter application. Kidney Int. 2002;61(1):133-140.

56. Cohen CD, et al. Comparative promoter analysis allows de novo identification of specialized cell junction-associated proteins. Proc Natl Acad Sci USA. 2006;103(15):5682-5687.

57. Tusher VG, Tibshirani R, Chu G. Significance analysis of microarrays applied to the ionizing radiation response. Proc Natl Acad Sci USA. 2001;98(9):5116-5121.

58. Buch T, et al. A Cre-inducible diphtheria toxin receptor mediates cell lineage ablation after toxin administration. Nat Methods. 2005;2(6):419-426.

59. Boerries M, et al. Molecular fingerprinting of the podocyte reveals novel gene and protein regulatory networks. Kidney Int. 2013;83(6):1052-1064

60. Weibel ER, Gomez DM. A principle for counting tissue structures on random sections. J Appl Physiol. 1962;17:343-348.

61. Schindelin J, et al. Fiji: an open-source platform for biological-image analysis. Nat Methods. 2012;9(7):676-682.

62. Kabgani N, et al. Primary cultures of glomerular parietal epithelial cells or podocytes with proven origin. PLoS ONE. 2012;7(4):e34907.

63. Shved N, et al. Transcriptome-based network analysis reveals renal cell type-specific dysregulation of hypoxia-associated transcripts. Sci Rep. 2017;7(1):8576. 